\title{
THETA FUNCTIONS AND ABELIAN VARIETIES OVER VALUATION FIELDS OF RANK ONE 1 .
}

\author{
HISASI MORIKAWA
}

Introduction. We shall denote by $\mathfrak{M}$ the $Z$-module of integral vectors of dimension $r$, by $T$ a symmetric complex matrix with positive definite imaginary part and by $z$ the variable vector. If we put $q(\mathrm{~m}, \mathfrak{n})=e^{\pi v-\overline{1} l \mathfrak{m} r \mathfrak{n}}$ and $u(\mathfrak{m})$

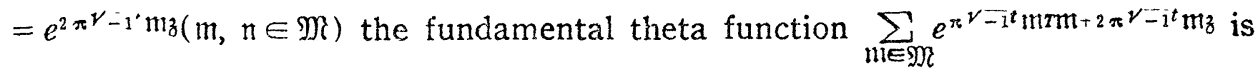
expressed in the form: $\vartheta(q \mid u)=\sum_{m \in \mathfrak{M}} q(\mathrm{~m}, \mathfrak{m}) u(\mathrm{~m})$ as a series in $q$ and $u$. Other theta functions in the classical theory are derived from the fundamental theta function by translating the origin and making sums and products, so these theta functions are also expressed in the form: $\sum_{m \in \mathfrak{m}} c_{\mathfrak{m}} q\left(\mathrm{~m}, n^{-1} \mathrm{~m}\right) \boldsymbol{u}(\mathrm{m})$ as series of $q$ and $u$. Moreover the coefficients in the relations of theta functions are also expressed in the form: $\sum_{\mathfrak{m} \in \mathfrak{M}} d_{\mathfrak{m}} g\left(\mathfrak{m}, n^{-1} \mathfrak{m}\right)$ as series in $q$.

All the parts of theory of theta functions are formal except only one point: The products of theta functions are also theta functions. This property of the products of theta functions comes out as a result from the positive definiteness of the imaginary part of $T$. The positive definiteness of the imaginary part of $T$ is equivalent to the condition: $|q(\mathfrak{m}, \mathrm{m})|<1$ for $\mathrm{m} \neq 0$.

This situation suggests the possibility of replacement of the field of complex numbers with a field complete with respect to a valuation of rank one.

\section{$\S 1$. Summary and notations.}

1.1. We mean by a valuation $v$ of rank one of a field $\Omega$ a mapping $v$ of $\Omega$ into the additive group of real numbers satisfying $v(\xi \eta)=v(\xi)+v(\eta)$ and $v(\xi+\eta) \geq \min \{v(\xi), v(\eta)\}(\xi, \eta \neq 0$ in $\Omega)$.

We fix, once for all in the following, an algebraically closed field $\Omega$ complete with respect to a (non-trivial) valuation $v$ of rank one. We also choose a field $\mathrm{K}$ containing $\Omega$ such that $1^{\circ} \mathrm{K}$ is algebraically closed and $2^{\circ} \mathrm{K}$ contains infinite many elements $x_{1}, x_{2}, \ldots$ such that there exists no relation $\sum_{i_{1}, \ldots, i_{r} \in n^{-1} Z} a_{i_{1} \cdots i_{r}} x_{1}^{i_{1}}$

Received October 20, 1961. 
$\cdots x_{r}^{i_{r}}=0\left(a_{i_{2} \cdot i_{r}} \in \Omega, n \in Z\right)$; we call $\mathrm{K}$ the univarsal domain of $\Omega$. We fix, once for all in the following, a mapping of $\mathrm{K}^{\times} \times \mathrm{Q}$ onto $\mathrm{K}^{\times}:(\xi, a) \rightarrow \xi^{a}\left(\mathrm{~K}^{\times}\right.$ $=\mathrm{K}-\{0\} ; \mathrm{Q}$ is the field of rational numbers) satisfying $1^{\circ} \xi^{a} \eta^{a}=(\xi \eta)^{a}, 2^{\circ} \xi^{a} \xi^{b}$ $=\xi^{a+b}, 3^{\circ}\left(\xi^{a}\right)^{b}=\xi^{a b}, 4^{\circ} \xi^{0}=1,5^{\circ} \xi^{n}=\xi \overbrace{}^{n} \xi \xi, 6^{\circ} 1^{m^{-1}}$ is a primitive $m_{0}$-th root of unity $\left(m=p^{\nu} m_{0} ;\left(m_{0}, p\right)=1\right)\left(\xi, \eta \in \mathrm{K}^{\times} ; a, b \in \mathrm{Q} ; m, n, m_{0} \in Z\right)$. By the Zorn's Lemma we can always construct a mapping $(\hat{\xi}, a) \rightarrow \xi^{i}$ satisfying $1^{\circ}, \ldots, 6^{\circ}$. By this mapping $\Omega$ is maped onto itself.

We denote by $\mathfrak{M}_{Q}$ a space of $r$-dimensional rational column vectors $\left\{{ }^{t}\left(x_{1}\right.\right.$, $\left.\left.\ldots, x_{r}\right) \mid x_{1}, \ldots, x_{r} \in \mathrm{Q}\right\}$ and denote by $\mathfrak{X}$ the $\mathrm{Z}$-module of all the integral column vectors in $\mathfrak{M}_{Q}$. For a $r$-square rational matrix $\alpha$ we mean by $\alpha \mathfrak{M}$ the $Z$-module $\{\alpha \mathfrak{m} \mid \mathfrak{m} \in \mathfrak{M}\}$.

In the following we shall fix once for all a positive definite symmetric integral matrix $S$ of degree $r$ and an involutive $Q$-algebra $\Lambda_{Q}$ in the full matrix ring $\operatorname{End}_{Q}\left(\mathfrak{M}_{Q}\right)$ with the involution $*: \alpha^{*}=S^{t} \alpha S^{-1}$, where we assume that $A_{Q}$ contains the identity matrix. We denote by $A$ the $Z$-algebra consisting of all the elements $\alpha$ such that $\alpha S^{-1} \mathfrak{M} \subset S^{-1} \mathfrak{M}$. An element $\alpha$ in $\Lambda_{\mathrm{Q}}$ is called symmetric if $\alpha^{*}=\alpha$ and a symmetric element $\beta$ is called positive if all the characteristic roots of $\beta$ are real positive.

We choose a particular Z-base $\left(m_{1}, \ldots, m_{r}\right)$ of $S^{-1} \mathfrak{M}$ such that $S m_{1}={ }^{t}(1,0$, $\ldots, 0), \ldots, S m_{r}={ }^{t}(0, \ldots, 0,1) ;\left(m_{1}, \ldots, m_{r}\right)$ is also a $Q$-base of $\mathfrak{M}_{Q}$. We write each vector $m$ in $\mathfrak{M}_{Q}$ with components $x_{1}(\mathfrak{m}), \ldots, x_{r}(\mathfrak{m})$ with respect to $\left(m_{1}, \ldots, m_{r}\right)$ as follows : $m=\sum_{i=1}^{r} x_{i}(m) m_{i}$.

1. 2. A function $\%$ on $\mathfrak{M}_{Q}$ valued in $\mathrm{K}^{\times}$is said multiplicative if $\%$ satisfies $\chi(m+n)=\chi(m) \chi(n)$ and a function $\psi$ on $\mathfrak{M}_{Q} \times \mathfrak{M}_{Q}$ is said bimultiplicative if $\psi\left(\mathfrak{m}+\mathfrak{m}^{\prime}, \mathfrak{n}+\mathfrak{n}^{\prime}\right)=\psi(\mathfrak{m}, \mathfrak{n}) \psi\left(\mathfrak{m}^{\prime}, \mathfrak{n}\right) \psi\left(\mathfrak{m}, \mathfrak{n}^{\prime}\right) \psi\left(\mathfrak{m}^{\prime}, \mathfrak{n}^{\prime}\right)\left(\mathfrak{m}, \mathfrak{m}^{\prime}, \mathfrak{n}, \mathfrak{n}^{\prime} \in \mathfrak{M}_{\mathbf{Q}}\right)$. If $\phi(\mathfrak{m}$, $\mathfrak{n})=\phi(\mathfrak{n}, \mathfrak{m}), \phi$ is called symmetric. If a symmetric $\phi$ has values in $\Omega^{\times}$and $v(v(m, m))>0$ for every non-zero $m$ in $\mathfrak{M}_{\mathrm{Q}}$, we call $\psi$ positive symmetric. All the multiplicative functions on $\mathfrak{M}_{Q}$ valued in $\mathrm{K}^{\times}$form a commutative group in the natural way; We denote it by $(F(K)$ and denote by $E(L)$ the subgroup of $\mathfrak{E}(\mathrm{K})$ consisting of all the multiplicative functions on $\mathfrak{M}_{Q}$ valued in a subfield $L$ of $\mathrm{K}$. A multiplicative function is uniquely determined by the system of values $\left(\%\left(\mathrm{~m}_{1}\right), \ldots, \gamma\left(\mathrm{m}_{r}\right)\right)$, because $\xi^{a}\left(\xi \in \mathrm{K}^{\times}, a \in \mathrm{Q}\right)$ are definite elements in $\Omega^{\times}$. So we may identify $[(L)$ with the direct product $L^{\times} \times \overbrace{\cdots \times}^{r} \bar{L}^{\times}$. Similarly 
a symmetric bimultiplicative function $\phi$ on $\mathfrak{R}_{Q}$ valued in $\Omega^{\times}$is als $n$ uniquely determined by $\psi\left(\mathrm{m}_{i}, \mathrm{~m}_{j}\right),(1 \leq i \leq j \leq \boldsymbol{r})$.

The full matrix ring $\operatorname{End}_{\mathrm{Q}}\left(\mathfrak{M}_{\mathrm{Q}}\right)$ operates on $\mathfrak{E}(\mathrm{K})$ in the following way: $\varkappa^{\alpha}(m)=\%(\alpha m),\left(\alpha \in \operatorname{End}_{Q}\left(\mathfrak{M}_{Q}\right), \psi \in\left(\xi(K), \mathfrak{m} \in \mathfrak{M}_{\mathrm{Q}}\right)\right.$.

Using multiplicative functions, we can characterize the universal domain $\mathrm{K}$ by the following conditions: $1^{\circ} \mathrm{K}$ is an algebraic closed field containing $\Omega$ and $2^{\circ}$ there exists infinite many elements $w_{1}, w_{2}, \ldots$ in $(\xi)(\mathrm{K})$ such that there

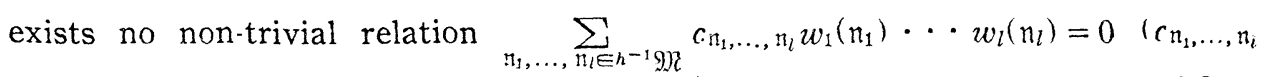
$\in \Omega ; h \in Z)$. We say $w_{1}, \ldots, w_{l}$ in $(\xi(\mathrm{K})$ to be independent over a subfield $L$ of $\mathrm{K}$ if there exists no non-trivial relation $\sum_{\left.n_{1}, \ldots, n_{l} \in h^{-1} \mathfrak{M}\right)} c_{\mathfrak{n}_{1}, \ldots, \mathfrak{n}_{l}} w_{1}\left(\mathfrak{n}_{1}\right) \cdots w_{l}\left(\mathfrak{n}_{l}\right)$ $=0 \quad\left(c_{n_{1}, \ldots, n_{l}} \in L ; h \in Z\right)$. If $w$ in $(E(K)$ satisfies no non-trivial relation: $\sum_{\mathfrak{n} \in h^{-1} \mathfrak{M}} c_{\mathfrak{n}} w(\mathfrak{n})=0(c \in L ; h \in Z)$, then we call $w$ a variable element in $(\mathfrak{F}(\mathrm{K})$ over $L$.

1.3. We choose a positive symmetric bimultiplicative function $q$ on $\mathfrak{M}_{Q}$ valued in $\Omega^{\times}$; Namely we choose a system $q\left(m_{i}, m_{j}\right)(1 \leq i \leq j \leq r)$ of elements in $\Omega^{\times}$such that $\left(v\left(q\left(\mathrm{~m}_{i}, \mathrm{~m}_{j}\right)\right)\right)$ is a positive definite real matrix. We denote by $q(\mathfrak{m})\left(\mathfrak{m} \in \mathfrak{M}_{Q}\right)$ the multiplicative functions defined by

(1) $\quad q(\mathfrak{m})(\mathfrak{n})=q(\mathfrak{m}, \mathfrak{n})^{2}\left(\mathfrak{n} \in \mathfrak{M}_{\mathbb{Q}}\right)$;

and we denote by $1(\mathfrak{m})\left(\mathfrak{m} \in \mathfrak{M}_{\mathbb{Q}}\right)$ the multiplicative function defined by

(2) $1(\mathfrak{m})(\mathfrak{n})=1(\mathfrak{m}, \mathfrak{n})=1^{t \mathfrak{m} S^{2} \mathfrak{n}}$.

Let $u_{1}, \ldots, u_{r}$ be indeterminates over $\mathrm{K}$. We define a multiplicative function $u$ on $\mathfrak{M}_{Q}$ as follows:

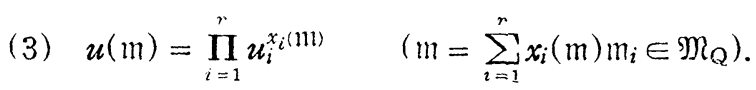

We shall now define theta functions and abelian functions:

Definition 1. A matrix $\alpha$ in $\operatorname{End}_{h}\left(S^{-1} \mathfrak{M}\right)$ is called a multiplication of a period $\left(S^{-1}, q\right)$ if $q\left(\alpha^{*} m, \mathfrak{n}\right)=q(\mathfrak{m}, \alpha \mathfrak{n})$ for $\mathfrak{m}, \mathfrak{n} \in \mathfrak{M}_{\mathrm{Q}}$, where $\alpha^{*}=S^{t} \alpha S^{-1}$. All the multiplications of $\left(S^{-1}, q\right)$ form a $Z$-algebra; We denote it by $\mathfrak{R}\left(S^{-1}, q\right)$.

Definition 2. Let $\alpha$ be a positive symmetric element in $\mathfrak{x}\left(S^{-1}, q\right)$ and $\chi$ be a multiplicative function on $\mathfrak{M}_{Q}$ valued in $\mathrm{K}$. A formal power series $\varphi(u)$ $=\sum_{m \in S^{-1} \mathfrak{M}} c_{\mathfrak{m}} u(\mathrm{~m})$ in $u_{1}, \ldots, u_{r}, u_{1}^{-1}, \ldots, u_{r}^{-1}$ is called a theta function of type 
$(\alpha, \chi)$ with period $\left(S^{-1}, q\right)$, if $\varphi(u)$ satisfies

(4) $\varphi(q(\mathrm{~m}) u)=q(\mathrm{~m}, \alpha \mathrm{m})^{-1} u(\alpha \mathrm{m})^{-1} \%(\alpha \mathrm{m})^{-1} \varphi(u),\left(\mathfrak{m} \in S^{-1} \mathfrak{M}\right)$,

where $q(\mathrm{~m}) u$ means the product of multiplicative functions $q(\mathrm{~m})$ and $u$. If all the coefficients $c_{\mathfrak{m}}\left(\mathfrak{m} \in S^{-1} \mathfrak{M}\right)$ belong to a field $L$, we call $\varphi(u)$ a theta function with coefficients in $L$.

Definition 3. The quotient $f(u)=\varphi(u) / \psi(u)$ of theta functions $\varphi(u), \psi(u)$ of the same type $(n, \chi)$ with period $\left(S^{-1}, q\right)$ is called an abelian function with period $\left(S^{-1}, q\right)$, where we restrict $n$ in the positine integers (See Proposition 12). If we can choose $\varphi(u)$ and $\phi(u)$ whose coefficients belong to $L$, we call $f(u)$ an abelian function with coefficients in $L$.

Since we shall see, in the following, that products of theta functions are also theta functions, the quotient $\varphi(u) / \psi(u)$ in Definition 3 has definite meaning.

In the present paper we shall prove the following results:

$1^{\circ}$ We denote by $\mathfrak{R}_{\mathfrak{2}}(\alpha, \chi)$ the vector space of theta functions of type $(\alpha, \%)$ with coefficients in $\Omega$. Then we have

$$
\operatorname{dim}_{\Omega} \mathfrak{M}_{\Omega}(\alpha, \chi)=\operatorname{det} \alpha \text { for } \chi \in \mathbb{E}(\Omega)
$$

$2^{\circ}$ A theta function in $\mathfrak{M}_{\Omega}(\alpha, \%)$ converges at every point $u_{1}=\xi_{1}, \ldots, u_{r}$ $=\xi_{r},\left(\xi_{1}, \ldots, \xi_{r} \in \Omega^{\times}\right)$.

$3^{\circ}$ All the abelian functions with the period $\left(S^{-1}, q\right)$ and with coefficients in $\Omega$ form a field $K_{S, q}$ such that $K_{s, q} / \Omega$ is separably and finitely generated.

$4^{\circ}$ A period $\left(S^{-1}, q\right)$ uniquely determines an abelian variety $A_{s, q}$ defined over $\Omega$ such that the law of composition of $A_{s, q}$ is induced from the composition in $\left(E(K)\right.$ and the field of rational functions on $A_{s, q}$ over $\Omega$ is canonically isomorphic to $K_{s, q}$.

$5^{\circ}$ There exists a system of $l$-adic (resp. $p$-adic) coordinates of $A_{s, q}$ and an isomorphism $\gamma$ of $\mathfrak{Y}\left(S^{-1}, q\right)$ into the ring of endomorphisms $\operatorname{End}_{z}\left(A_{s, q}\right)$ of $A_{s, q}$ such that

$$
M_{l}(\gamma(\alpha))=\left(\begin{array}{cc}
S \alpha S^{-1} & 0 \\
0 & S \alpha S^{-1}
\end{array}\right)
$$

and, if $p \neq 0$,

$$
M_{p}(\gamma(\alpha))=S \alpha S^{-1} \quad\left(\alpha \in \mathfrak{R}\left(S^{-1}, q\right)\right)
$$


where $M_{l}$ (resp. $\left.M_{p}\right)$ is the $l$-adic (resp. $p$-adic) representation of End $\left(A_{s, q}\right)$ by the system of $l$-adic (resp. $p$-adic) coordinates.

$6^{\circ}$ Let $\Lambda_{Q}^{c}$ be the commutor algebra of $\Lambda_{Q}$ in $\operatorname{End}_{Q}\left(\mathfrak{M}_{Q}\right)$, i.e. the Q-subalgebra $\left\{\beta \in \operatorname{End}_{Q}\left(\mathfrak{M}_{Q}\right) \mid \alpha \beta=\beta \alpha\right.$ for every $\alpha$ in $\left.A_{Q}\right\}$. Then $\Lambda_{Q}^{c}$ has also the involution *: $\alpha^{*}=S^{t} \alpha S^{-1}$. If $A_{\mathrm{Q}}^{c}$ is generated by symmetric elements in $A_{\mathrm{Q}}^{c}$, then there exists a positive symmetric bimultiplicative function $q$ on $\mathfrak{M}_{\mathbf{Q}} \times \mathfrak{M}_{\mathrm{Q}}$ valued in $\Omega^{\times}$such that the ring of multiplications $\mathfrak{A}\left(S^{-1}, q\right)$ of $\left(S^{-1}, q\right)$ coincides with $A$.

\section{§2. Formal theta functions.}

2. 1. Let $\mathfrak{Q}$ be a symbolic symmetric bimultiplicative function on $\mathfrak{M}_{Q} \times \mathfrak{M}_{Q}$, i.e. $\mathfrak{Q}$ is a symbol satisfying the following relations:

(5) $\mathfrak{Q}\left(m+\mathfrak{n}, \mathfrak{m}^{\prime}+\mathfrak{n}^{\prime}\right)=\mathfrak{Q}\left(\mathfrak{m}, \mathfrak{m}^{\prime}\right) \mathfrak{Q}\left(m, \mathfrak{n}^{\prime}\right) \mathfrak{Q}\left(\mathfrak{n}, \mathfrak{m}^{\prime}\right) \mathfrak{Q}\left(\mathfrak{n}, \mathfrak{n}^{\prime}\right)$

(6) $\mathfrak{Q}(\mathfrak{m}, \mathfrak{n})=\mathfrak{L}(\mathfrak{n}, \mathfrak{m}),\left(\mathfrak{m}, \mathfrak{m}^{\prime}, \mathfrak{n}, \mathfrak{n}^{\prime} \in \mathfrak{M}_{Q}\right)$.

Furthermore we assume

$$
\mathfrak{Q}\left(\alpha^{*} \mathrm{~m}, \mathfrak{n}\right)=\mathfrak{Q}(\mathfrak{m}, \alpha \mathfrak{n})
$$

$$
\left(\mathfrak{m}, \mathfrak{n} \in \mathfrak{M}_{Q} ; \alpha \in \Lambda\right) .
$$

We call $\mathfrak{Q}$ the symbolic bimultiplicative function associated with $S$ and 1 . Later we shall specialize $\mathfrak{Q}$ to a positive symmetric bimultiplicative function $q$ valued in $\Omega^{\times}$.

We define the multiplicative function $\mathscr{Q}(m)$ by $\mathscr{L}(m)(\mathfrak{n})=\mathscr{L}(\mathrm{m}, \mathfrak{n})^{2},(\mathrm{~m}, \mathfrak{n}$ $\in \mathfrak{M}_{\mathrm{Q}}$ ).

The formal theta function is defined by

(8) $\vartheta(\mathfrak{Q} \mid u)=\sum_{\mathfrak{m} \in S^{-1} \mathfrak{m}} \mathfrak{Q}(\mathrm{m}, \mathrm{m}) u(\mathrm{~m})$.

For vectors $\mathfrak{g}, \mathfrak{h}$ in $\mathfrak{M}_{\mathrm{Q}}$ we shall define the formal theta function with characteristic $[\mathfrak{g}, \mathfrak{h}]$ as follows :

(9) $\vartheta[\mathfrak{g}, \mathfrak{h}](\mathfrak{D} \mid u)=\sum_{\mathfrak{m} \in S^{-1} \mathfrak{M}} \mathfrak{Q}(\mathfrak{m}+g, \mathfrak{m}+g) u(\mathfrak{m}+g) 1(\mathfrak{h}, \mathfrak{m}+g)$.

Then, by simple culculation, we have the following formulae:

(10) $\mathfrak{l}[\mathfrak{g}, \mathfrak{h}](\mathfrak{D} \mid u)=\mathfrak{g}(\mathfrak{D} \mid \mathfrak{S}(\mathfrak{g}) 1(\mathfrak{h}) u) \subseteq(g, g) u(\mathfrak{g}) 1(\mathfrak{h}, \mathfrak{g})$,

(11) $\mathfrak{g}[\mathfrak{g}, \mathfrak{h}](\mathfrak{D} \mid \mathfrak{I}(\mathrm{m}) 1(\mathfrak{n}) u)$

$$
=E(m, m)^{-1} u(m)^{-1} 1(\mathfrak{g}, \mathfrak{n}) 1(\mathfrak{h},-m) \vartheta[\mathfrak{g}, \mathfrak{h}](\mathfrak{D} \mid u),\left(\mathfrak{m}, \mathfrak{n} \in S^{-1} \mathfrak{M}\right),
$$


(12) $\vartheta[\mathfrak{g}, \mathfrak{h}]\left(\mathfrak{Q} / \mathfrak{Q}\left(\mathfrak{g}^{\prime}\right) 1\left(\mathfrak{h}^{\prime}\right) u\right)$

$$
=\vartheta\left[\mathfrak{g}+\mathfrak{g}^{\prime}, \mathfrak{h}+\mathfrak{h}^{\prime}\right](\mathfrak{Q} \mid u) \mathfrak{Q}\left(\mathfrak{g}^{\prime}, \mathfrak{g}^{\prime}\right)^{-1} u\left(g^{\prime}\right)^{-1} 1\left(\mathfrak{g}^{\prime}, \mathfrak{h}+\mathfrak{h}^{\prime}\right)^{-1},\left(\mathfrak{g}^{\prime}, \mathfrak{h}^{\prime} \in \mathfrak{M}_{\mathbf{Q}}\right) .
$$

(13) $\vartheta[\mathfrak{g}+\mathfrak{m}, \mathfrak{h}+\mathfrak{n}](\mathfrak{Q} \mid u)=\vartheta[\mathfrak{g}, \mathfrak{h}](\mathfrak{Q} \mid u) 1(\mathfrak{g}, \mathfrak{n}),\left(\mathfrak{m}, \mathfrak{n} \in S^{-1} \mathfrak{M}\right)$,

(14) $\vartheta[\mathfrak{g}, \mathfrak{h}](\mathfrak{Q} \mid u)=\vartheta[-\mathfrak{g},-\mathfrak{h}](\mathfrak{Q} \mid u)$.

The formal theta function of order $\alpha$ ( $\alpha$ is a positive symmetric element in $A$ ) with characteristic $[\mathfrak{g}, \mathfrak{h}]$ is defined as follows :

$$
\begin{aligned}
\vartheta_{\alpha}[\mathfrak{g}, \mathfrak{h}] & (\mathfrak{Q} \mid u) \\
= & \sum_{\mathfrak{m} \in S^{-1} \mathfrak{M}} \mathfrak{Q}(\mathfrak{m}+g, \alpha(\mathfrak{m}+g)) u(\alpha(\mathfrak{m}+g)) 1(h, \alpha(m+g)) .
\end{aligned}
$$

Then, by simple calculation, we have

(16) $\vartheta_{\alpha}[g, \mathfrak{h}](\mathfrak{Q} \mid \mathfrak{Q}(\mathrm{m}) 1(\mathfrak{n}) u)$

$$
\begin{array}{r}
=\mathscr{Q}(\mathfrak{m}, \alpha \mathfrak{m})^{-1} u(\alpha \mathfrak{m})^{-1} 1(\mathfrak{n}, \alpha \mathfrak{g}) 1(\mathfrak{h},-\alpha \mathfrak{m}) \vartheta_{\alpha}[g, \mathfrak{h}](\mathbb{Q} \mid u) \\
\left(\mathfrak{m}, \mathfrak{n} \in S^{-1} \mathfrak{M}\right) .
\end{array}
$$

For symbolic $\mathfrak{Q}$ we shall define a theta function of type $(\alpha, \%)$ as a power series $\varphi(u)=\sum_{\mathfrak{m} \in S^{-1} \mathfrak{M}} c_{\mathfrak{m}} u(\mathfrak{m})$ in $u_{\mathfrak{i}}, \ldots, u_{r}, u_{1}^{-1}, \ldots, u_{r}^{-1}$ with coefficients in a field such that $\varphi(\mathfrak{Q}(\mathrm{m}) u)=\mathfrak{Q}(\mathfrak{m}, \alpha \mathfrak{m})^{-1} u(\alpha m)^{-1} \chi(\alpha m)^{-1} \varphi(u)\left(m \in S^{-1} \mathfrak{M}\right)$.

We denote by $\left\{g_{1}=0, g_{2}, \ldots, g_{d(\alpha)}\right\}$ a system of representatives of $\alpha^{-1} S^{-1} \mathfrak{M} / S^{-1} \mathfrak{M}$ in $\alpha^{-1} S^{-1} \mathfrak{M}$, where $d(\alpha)=\operatorname{det} \alpha$. We shall now prove that $\left\{\vartheta_{\alpha}\left[g_{1}, 0\right](\mathfrak{Q} \mid \% u), \ldots, \vartheta_{\alpha}\left[g_{d(\alpha)}, 0\right](\mathfrak{Q} \mid \% u)\right\}$ form a base of theta functions of type $(\alpha, \gamma)$.

Proposition 1. Let $\varphi(u)=\sum_{\mathfrak{m} \in S^{-1} \mathfrak{M}} c_{\mathfrak{m}} u(m)$ be a formal theta function of type $(\alpha, \chi)$. Then we have

$$
\begin{aligned}
& \text { (17) } \begin{aligned}
\mathfrak{Q}\left(\mathrm{m}+\mathrm{g}_{i}, \alpha\left(\mathrm{m}+\mathrm{g}_{i}\right)\right)^{-1} \chi\left(\alpha\left(\mathrm{m}+\mathrm{g}_{i}\right)\right)^{-1} c_{\alpha\left(\mathfrak{m}+\mathrm{g}_{i}\right)} \\
=\mathfrak{Q}\left(g_{i}, \alpha g_{i}\right)^{-1} \chi\left(\alpha g_{i}\right)^{-1} c_{\alpha} g_{i}, \quad\left(m \in S^{-1} \mathfrak{M}\right),
\end{aligned} \\
& \text { (18) } \varphi(u)=\sum_{i=1}^{\alpha(\alpha)} \mathfrak{Q}\left(g_{i}, \alpha g_{i}\right)^{-1} \psi\left(\alpha g_{i}\right)^{-1} c_{\alpha} g_{i} \vartheta_{\alpha}\left[g_{i}, 0\right](\mathfrak{Q} \mid \% u) .
\end{aligned}
$$

Proof. For any element $\mathfrak{n}$ in $S^{-1} \mathfrak{M}$ we have

$$
\begin{aligned}
& \varphi(\mathfrak{Q}(\mathfrak{n}) u)=\sum_{\mathfrak{m} \in S^{-1} \mathfrak{M}} c_{\mathfrak{l}} \mathfrak{Q}(\mathfrak{n}, \mathfrak{m})^{2} u(\mathfrak{m}) \\
& =\sum_{i=1}^{a(\alpha)} \sum_{\mathfrak{m} \in S^{-1} \mathfrak{M} i} c_{\alpha\left(\mathfrak{m}+\mathfrak{g}_{i}\right)} \mathfrak{Q}\left(\mathfrak{n}, \alpha\left(\mathfrak{m}+\mathfrak{g}_{l}\right)\right)^{2} u\left(\alpha\left(\mathfrak{m}+g_{i}\right)\right) \\
& \left.=\mathfrak{Q}(\mathfrak{n}, \alpha \mathfrak{n})^{-1} \chi(\alpha \mathfrak{n})^{-1} u(\alpha \mathfrak{n})^{-1} \sum_{i=1}^{\alpha(\alpha)} \sum_{\mathfrak{m} \in S^{-1} \mathfrak{M} \mathbb{\alpha}} c_{\alpha\left(\mathfrak{m}+\mathfrak{g}_{i}\right.}\right) u\left(\alpha\left(\mathfrak{m}+\mathfrak{g}_{i}\right)\right) \text {. }
\end{aligned}
$$


This shows

$$
\mathfrak{Q}(\mathfrak{n}, \alpha \mathfrak{n})^{-1} \%(\alpha \mathfrak{n})^{-1} c_{\alpha\left(\mathfrak{m}+\mathfrak{n}+\mathfrak{g}_{i}\right)}=\mathfrak{E}\left(\mathfrak{n}, \alpha\left(\mathfrak{m}+\mathfrak{g}_{i}\right)\right)^{2} c_{\alpha\left(\mathfrak{m}+\mathfrak{g}_{i}\right)} .
$$

Putting $m=0$, we have

$$
\mathfrak{I}(\mathfrak{n}, \alpha \mathfrak{n})^{-1} \not(\alpha \mathfrak{n})^{-1} c_{\alpha\left(\mathfrak{n}+g_{i}\right)}=\mathbb{I}\left(\mathfrak{n}, \alpha g_{i}\right)^{2} c_{\alpha\left(\mathfrak{n}+g_{i}\right)} .
$$

Since $\alpha^{*}=\alpha$ and $\mathfrak{Q}\left(\alpha^{*} m, \mathfrak{n}\right)=\mathfrak{D}(\mathfrak{m}, \alpha \mathfrak{n})$, it follows

$$
\begin{aligned}
& \mathfrak{Q}\left(\mathfrak{n}+g_{i}, \alpha\left(\mathfrak{n}+\mathfrak{g}_{i}\right)\right)^{-1} \%\left(\alpha\left(\mathfrak{n}+\mathfrak{g}_{i}\right)\right)^{-1} c_{\alpha\left(\mathfrak{n}+\mathfrak{g}_{i}\right)} \\
& =\mathscr{S}(\mathfrak{n}, \alpha \mathfrak{n})^{-1} \Xi\left(\mathfrak{n}, \alpha g_{i}\right)^{-2} \Omega\left(g_{i}, \alpha g_{l}\right)^{-1} \%(\alpha \mathfrak{n})^{-1} \%\left(\alpha g_{i}\right)^{-1} c_{\alpha\left(\mathfrak{n}+g_{i}\right)} \\
& =\operatorname{E}\left(g_{i}, \alpha g_{i}\right)^{-1} \%\left(\alpha g_{i}\right)^{-1} c_{\alpha} g_{i} \text {. }
\end{aligned}
$$

This implies

$$
\begin{gathered}
\varphi(u)=\sum_{\mathfrak{m} \in S^{-1} \mathfrak{M}} c_{\mathfrak{m}} u(\mathfrak{m})=\sum_{i=1}^{\alpha(\alpha)} \sum_{\mathfrak{m} \in S^{-1} \mathfrak{M}} \mathfrak{Q}\left(\alpha\left(\mathfrak{m}+g_{i}\right), \mathfrak{m}+g_{i}\right) \%\left(\alpha\left(\mathfrak{m}+g_{i}\right)\right) \\
\mathscr{E}\left(g_{i}, \alpha g_{i}\right)^{-1} \%\left(\alpha g_{i}\right)^{-1} c_{\alpha \mathfrak{g}_{i}} u\left(\alpha\left(\mathfrak{m}+g_{i}\right)\right) \\
=\sum_{i=1}^{\alpha(\alpha)} \mathfrak{Q}\left(g_{i}, \alpha g_{i}\right)^{-1} \%\left(\alpha g_{i}\right)^{-1} c_{\alpha g_{i}} \vartheta_{\alpha}\left[g_{i}, 0\right](\mathfrak{D} \mid \% u) .
\end{gathered}
$$

Proposition 2. $\vartheta_{\alpha}\left[g_{1}, 0\right](\Xi \mid u), \ldots, \vartheta_{\alpha}\left[g_{d(\alpha)}, 0\right](\mathfrak{Q} \mid u)$ are linearly independent as series in $u$.

Proof. Since for $i \neq j$ there exists no element $m$ in $S^{-1} \mathfrak{M}$ satisfying $u\left(\alpha g_{i}\right)$ $=u\left(\alpha\left(g_{j}+m\right)\right)$. This shows that $\vartheta_{x}\left[g_{1}, 0\right](\mathfrak{D} \mid u), \ldots, \vartheta_{\alpha}\left[g_{d(\alpha)}, 0\right](\mathbb{Q} \mid u)$ are linearly independent as series in $u$.

Remark. This Proposition is also true for any specialization of $\mathbb{Q}$.

Proposition 3. Let $\alpha$ and $\beta$ be positive symmetric elements of $A$. If $\alpha$, $\beta g^{\prime} \in S^{-1} \mathfrak{M}$, then $\vartheta_{\alpha}[g, 0](\mathbb{D} \mid \% u) \vartheta_{\beta}\left[g^{\prime}, 0\right]\left(\mathbb{D} \mid \chi^{\prime} u\right)$ is a formal theta function of tvpe $\left(\alpha+\beta, \chi^{\alpha(\alpha+\beta)^{-1}} /^{\left(\beta(\alpha+\beta)^{-1}\right.}\right)$ given by the series:

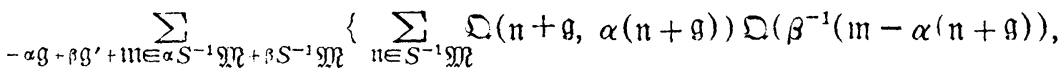

$$
\begin{aligned}
& \left.\mathfrak{m}-\alpha(\mathfrak{n}+\mathfrak{g})) \%(\alpha(\mathfrak{n}+\mathfrak{g})) \chi^{\prime}(\mathfrak{m}-\alpha(\mathfrak{n}+\mathfrak{g}))\right\} u(\mathfrak{m}) .
\end{aligned}
$$

'This is an immediate consequence from the expansions of $\vartheta_{\alpha}[g, 0](\mathbb{Q} \mid \boldsymbol{u})$ and $\vartheta_{\beta}\left[g^{\prime}, 0\right](\mathfrak{Q} \mid u)$. This proposition is also true for any specialization $\Sigma^{\prime}$ of $\mathfrak{Q}$ provided that

$$
\begin{array}{r}
\sum_{r \in S^{-1} \mathfrak{M}} \mathbb{Q}^{\prime}(\mathfrak{n}+g, \alpha(\mathfrak{n}+g)) \mathfrak{S}^{\prime}\left(\beta^{-1}(\mathfrak{m}-\alpha(\mathfrak{n}+\mathfrak{g})), \mathfrak{m}-\alpha(\mathfrak{n}+\mathfrak{g})\right) \\
\chi(\alpha(\overrightarrow{\mathfrak{n}}+g)) \varkappa^{\prime}(\mathfrak{m}-\alpha(\mathfrak{n}+\mathfrak{g}))
\end{array}
$$


has definite meaning.

By virtue of (18) and Proposition 3 we have

Proposition 4. If $\varphi\left(u^{\prime}\right)$ and $\psi(u)$ are fornal theta functions of type $(\alpha, \%)$ and $(\beta, \%)$, respectively, then $\varphi(u) \psi(u)$ is a formal theta function of type $\left(\alpha+\beta, \%^{\alpha(\alpha+\beta)^{-1}} \%^{\beta(\alpha+\beta)^{-1}}\right)$.

Corollary. If $\varphi_{1}(u), \ldots, \varphi_{t}(u)$ are formal theta funstions of type $(\alpha, \gamma)$, then $\prod_{i=1}^{t} \varphi_{i}(u)$ is a formal theta function of type $(t \alpha, \%)$.

PROPOSITION 5. If $n$ is a positive integer greater than one, then there exists no homogeneous relations of $\vartheta_{n}[0,0](\mathbb{Q} \mid u), \vartheta_{n}\left[n^{-1} \mathrm{~m}_{1}, 0\right](\mathfrak{Q} \mid u), \ldots, \vartheta_{n}\left[n^{-1} \mathrm{~m}_{r}\right.$, $0](\mathbb{Q} \mid u)$ as functions in $u$.

Proof. We shall denote briefly $\varphi_{0}(u)=\vartheta_{n}[0,0](\mathcal{Q} \mid u), \varphi_{i}(u)=\vartheta_{n}\left[n^{-1} \mathfrak{m}_{i}\right.$, $0](\mathfrak{Q} \mid u)(1 \leq i \leq r)$. Assume, for a moment, there exists a non-trivial homogeneous relation of degree $d$

(*) $\sum_{\nu=1}^{t} a_{\left[\lambda_{\nu}\right]} \varphi_{i}^{\lambda_{\nu 0}} \cdots \varphi_{r}^{\lambda_{\nu r}}=0$,

where we may assume that the degree $d$ is the lowest value of the possible case and the number of terms $t$ is the least value provided that the degree is $d$. From the expansions of $\varphi_{i}(0 \leq i \leq r)$, each term of $\varphi_{0}^{\lambda_{\nu}} \cdots \varphi_{r}^{\lambda_{r r}}$ is of the following form:

$$
c_{\left.\left[\lambda_{l}\right] \mathfrak{h}\right)} u\left(n \sum_{l=0}^{r} \lambda_{\nu l} \mathfrak{h}^{(l)}+\sum_{j=1}^{r} \lambda_{v j} \mathfrak{m}_{j}\right) \quad\left(\mathfrak{h}^{(l)} \in S^{-1} \mathfrak{M}\right) .
$$

Therefore the degree of $u_{i}$ in each term of $\varphi_{0}^{\lambda_{v}} \cdots \varphi_{r}^{\lambda_{v r}}$ is of the following form :

$$
(* *) \quad n \sum_{l=0}^{r} \lambda_{\nu l} x_{i}\left(\mathfrak{G}^{(l)}\right)+\sum_{j=1}^{r} \lambda_{v j} \delta_{i j}=n \sum_{l=0}^{r} \lambda_{\nu l} x_{i}\left(\mathfrak{h}^{(l)}\right)+\lambda_{v i} .
$$

If $\lambda_{i i} \lambda_{i i}(\bmod n)$ for some $\nu$ and $\bar{\nu}$, the number of terms in

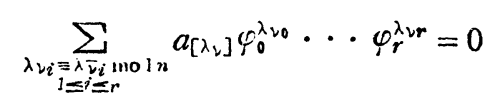

is smaller than $t$. This contradicts the assumption on $t$. Hence $\lambda_{\nu i} \equiv \lambda_{\nu^{\prime} i} \bmod n$ for every $\nu$ and $\nu^{\prime}$. Since the degree $d$ is lowest, $\lambda_{\nu i}(i=1,2, \ldots, r ; \nu=1,2$, $\ldots, t)$ must be congruent to zero $\bmod n$. From this it follows that $\lambda_{20} \equiv \lambda_{2,0}$ 
$\bmod n\left(\nu, \nu^{\prime}=1,2, \ldots, t\right)$. We denote by $f$ the positive integer such that $0 \leq f<n$ and $\lambda_{\nu 0}+f \equiv 0 \bmod n$. Putting $\psi_{i}(u)=\varphi_{i}^{n}(u)(i=0,1,2, \ldots, r)$, we have a relation of $\psi_{0}, \ldots, \psi_{r}$ of degree $d^{\prime}=(d+f) n^{-1}$

$(* * *) \quad \sum_{\nu=1}^{t} a_{\left[\lambda_{\nu}\right]} \psi_{0}^{\mu \nu \nu_{0}} \cdots \psi_{r}^{\mu \nu r}=0$, where

$$
\mu_{\nu 0}=n^{-1}\left(\lambda_{\nu 0}+f\right), \quad \mu_{\nu i}=n^{-1} \lambda_{v i} \quad(1 \leq i \leq r) .
$$

Then the degree $d^{\prime}$ is the lowest of the possible case and the number of terms $t$ is the least one. Moreover, by virtue of $(* *)$, the degree of $u_{i}$ in each term of $\psi_{0}^{\mu_{v 0}} \cdots \psi_{r}^{\mu_{v r}}$ is of the form

$$
n\left(n \sum_{l=0}^{r} \mu_{v l} x_{i}\left(\mathfrak{G}^{(l)}\right)\right)+n \mu_{v j} \delta_{i j}=n^{2} \sum_{l=0}^{r} \mu_{v l} x_{i}\left(\mathfrak{h}^{(l)}\right)+n \mu_{v i} .
$$

Therefore, by the same reason as for $\left\{\lambda_{\nu l}\right\}$, we have $\mu_{\nu 0}+f^{\prime} \equiv \mu_{\nu 1} \equiv \cdots \equiv \ddot{i \nu, r} \equiv 0$ $\bmod n$ with $f^{\prime}$ satisfying $0 \leq f^{\prime}<n$ and $d>d^{\prime}=(d+f) n^{-1}>d^{\prime \prime}=\left(d^{\prime}+f^{\prime}\right) n^{-1}$. This shows that the precess of generating $\left\{\mu_{v i}\right\}$ from $\left\{\lambda_{, i}\right\}$ does not stop. It is, however, impossible, for the degree $d$ of $(*)$ is finite. This is a contradiction.

2. 2. Let $\chi_{1}, \ldots, \chi_{t}$ be multiplicative functions on $\mathfrak{M}_{\mathrm{Q}}$. We shall denote by $Z\left[\left[\Omega, \chi_{1}, \ldots, \chi_{t}\right]\right]$ the $Z$-algebra generated by $\mathfrak{Q}(m, \mathfrak{n}), \chi(m),\left(m, \mathfrak{n} \in \mathfrak{M}_{\mathbb{Q}}\right)$ and series

$$
\begin{array}{r}
\sum_{\mathfrak{n} \in S^{-1} \mathfrak{M}} \mathfrak{Q}(\mathfrak{n}+\mathfrak{g}, \alpha(\mathfrak{n}+\mathfrak{g})) \mathfrak{Q}\left(\beta^{-1}(\mathfrak{m}-\alpha(\mathfrak{n}+\mathfrak{g})), \mathfrak{m}-\alpha(\mathfrak{n}+\mathfrak{g})\right) \%(\alpha(\mathfrak{n}+\mathfrak{g})) \\
\varkappa^{\prime}(\mathfrak{m}-\alpha(\mathfrak{n}+\mathfrak{g}))
\end{array}
$$

( $\alpha, \beta$ : positive symmetric elements in $A ; \mathfrak{g}, \mathfrak{m} \in \mathfrak{M}_{\mathrm{Q}}$;

$\chi, \chi^{\prime}$ : multiplicative functions $\left.\chi_{1}^{r_{1}} \ldots \gamma_{t}^{\Upsilon_{t}}\left(\gamma_{1}, \ldots, \gamma_{t} \in \Lambda_{Q}\right)\right)$.

We denote by $Q\left(\left(\Omega, \chi_{1}, \ldots, \chi_{t}\right)\right)$ the quotient field of $Z\left[\left[\Omega, \chi_{1}, \ldots, \chi_{t}\right]\right]$.

Proposition 6. Let $n$ be a positive integer greater than one and $\%$ be a multiplicative function on $\mathfrak{M}_{\mathrm{Q}}$. Put

$$
\begin{aligned}
& \varphi_{0}(u)=\vartheta\left(\mathfrak{D} \mid \chi^{n+1} u\right) \vartheta_{n}[0,0](\mathfrak{Q} \mid u), \\
& \varphi_{1}(u)=\vartheta\left(\mathfrak{Q} \mid \chi^{n+1} u\right) \vartheta_{n}\left[n^{-1} \mathrm{~m}_{1}, 0\right](\mathfrak{D} \mid u), \ldots, \\
& \varphi_{r}(u)=\dot{\vartheta}\left(\mathfrak{Q} \mid \chi^{n+1} u\right) \vartheta_{n}\left[n^{-1} \mathrm{~m}_{r}, 0\right](\mathfrak{Q} \mid u)
\end{aligned}
$$

(They are theta functions of type $(n+1, \%)$ ). Then there exists a positive integer $M(n)$ such that for any positive integer $m$ and any theta function $\psi$ of type $(m, \chi)$ with coefficients in $\mathrm{Q}((\mathcal{D}, \chi))$ there exists a homogeneous relation 
in $\psi, \varphi_{0}, \ldots, \varphi_{r}$ at most of degree $M(n)$ with coefficients in $Z[[\Omega, \chi]]$ which is non-trivial on $\psi$.

Proof. By virtue of Propositions 1 and 3, there exists a non-zero element $\xi$ in $Z[[\Omega, \chi]]$ such that the coefficients of $u(m)$ in $\xi \psi(u)\left(m \in S^{-1} \mathfrak{R}\right)$ belong to $Z[[Q, \%]]$. Since $\psi$ is a theta function of type $(m, \%)$ with coefficients in $\mathrm{Q}((\Omega, \chi))$, putting $\phi=\phi^{n+1}$, we get a theta function $\phi$ of type $((n+1) m, \chi)$. Since the number of products of degree $x$ of $\varphi_{0}, \ldots, \varphi_{r}$ equals to $\left(\begin{array}{c}x+r \\ r\end{array}\right)$, the number of products of $\phi, \varphi_{0}, \ldots, \varphi_{r}$ that are theta functions of type $(x(n$ $+1) m, \chi$ ) equals to

$$
\begin{aligned}
\left(\begin{array}{c}
x m+r \\
r
\end{array}\right)+ & \left(\begin{array}{c}
(x-1) m+r \\
r
\end{array}\right)+\cdots+1 \\
= & \frac{m^{r}}{r !}\left\{\left(x+\frac{r}{m}\right) \cdots\left(x+\frac{1}{m}\right)+\left(x-1+\frac{r}{m}\right)\right. \\
& \left.\cdots\left(x-1+\frac{1}{m}\right)+\cdots+\frac{r !}{m^{r}}\right\}
\end{aligned}
$$

If $x>(n+1)^{r} r$, this number is greater than

$$
m^{r}(r !)^{-1}\left\{x^{r}+(x-1)^{r}+\cdots+\left(x-(n+1)^{r} r !\right)^{r}\right\} .
$$

On the other hand, by virtue of Propositions 1 and 2, the number of linearly independent theta functions of type $(x(n+1) m, \chi)$ equals to $x^{r}(n+1)^{r} m^{r}$. Since the reading term of

$$
x^{r}+(x-1)^{r}+\cdots+\left(x-(n+1)^{r} r !\right)^{r}-r !(n+1)^{r} x^{r} \text { is } x^{r},
$$

there exists a positive integer $M$ such that

$$
M^{r}+(M-1)^{r}+\cdots+\left(M-(n+1)^{r} r !\right)^{r}-r !(n+1)^{r} M^{r}>0 .
$$

Hence we have

$$
\left(\begin{array}{c}
M m+r \\
r
\end{array}\right)+\left(\begin{array}{c}
(M-1) m+r \\
r
\end{array}\right)+\cdots+1>M^{r}(n+1)^{r} m^{r}
$$

for every $m>0$ in $Z$. This shows that there exists a non-trivial relation of degree $M$ and $M$ is independent of $m$. From Proposition $5, \varphi_{0}, \ldots, \varphi_{r}$ are inderendent, hence the relation is non-trivial on $\psi$. Since all the coefficients belong to $\mathfrak{Q}((\mathbb{Q}, \chi))$, the coefficients in the relation can be chosen in $Z[[\mathbb{Q}, \%]]$. Therefore, putting $M(n)=(n+1) M$, we get $M(n)$ in the Proposition. 
Corollary. Let $\varphi(u)$ and $\psi(u)$ be theta function of type $(m, \%)$ with coefficients in $\mathrm{Q}((\mathfrak{D}, \chi))$. Then there exists a homogeneous relation in $\varphi / \psi, \varphi_{1} / \varphi_{0}$, $\ldots, \varphi_{r} / \varphi_{0}$ at most of degree $M(n)^{2}$ which is non-trivial on $\varphi / \psi$.

Proof. By virtue of Proposition 6,

$\left[Q((\Omega, \chi))\left(\varphi / \varphi_{0}, \varphi_{1} / \varphi_{0}, \ldots, \varphi_{r} / \varphi_{0}\right):\right.$

$$
\left.\mathrm{Q}((\mathfrak{Q}, \chi))\left(\varphi_{1} / \varphi_{0}, \ldots, \varphi_{r} / \varphi_{0}\right)\right] \leq M(n)
$$

and

$\left[\mathrm{Q}((囚, \%))\left(\psi / \varphi_{0}, \varphi / \varphi_{0}, \ldots, \varphi_{r} / \varphi_{n}\right):\right.$

$$
\left.\mathrm{Q}((\Omega, \chi))\left(\varphi_{1}^{\prime} \varphi_{0}, \ldots, \varphi_{r} / \varphi_{0}\right)\right] \leq M(n) .
$$

Hence

$$
\begin{aligned}
& {\left[\mathrm{Q}((\mathfrak{Q}, \chi))\left(\varphi / \psi, \varphi_{1} / \varphi_{0}, \ldots, \varphi_{r} / \varphi_{0}\right):\right.} \\
& \mathrm{Q}\left((\mathfrak{Q}, \chi)\left(\varphi_{1} / \varphi_{0}, \ldots, \varphi_{r} / \varphi_{0}\right)\right] \leq M(n)^{2} .
\end{aligned}
$$

Proposition 7. Let $\varphi_{0}, \ldots, \varphi_{r+1}$ be theta functions of type $(\alpha, \%)$ with coefficients in $\mathrm{Q}((\mathfrak{Q}, \chi))$. Then there exists a non-trivial homogeneous relation in $\varphi_{0}, \ldots, \varphi_{r+1}$ with coefficients in $\mathrm{Q}((\Omega, \%))$.

Proof. The number of products of degree $x$ of $\varphi_{0}, \ldots, \varphi_{r+1}$ equals to $\left(\begin{array}{c}x+r+1 \\ r+1\end{array}\right)$ and the type of such products is $(x \alpha, \%)$. On the other hand the number of linearly independent formal theta functions of type $(x \alpha, \%)$ equals to $x^{r} \operatorname{det} \alpha$. Since the reading term of $\left(\begin{array}{c}x+r+1 \\ r+1\end{array}\right)$ is $((r+1) !)^{-1} x^{r+1}$, we have $\left(\begin{array}{c}M+r+1 \\ r+1\end{array}\right)>M^{r} \operatorname{det} \alpha$ for a sufficiently large $M$. This implies the existence of a non-trivial relation of $\varphi_{0}, \ldots, \varphi_{r+1}$. Since the coefficients in the products of $\varphi_{0}, \ldots, \varphi_{r+1}$ belong to $\mathrm{Q}((\mathbb{D}, \chi))$, the coefficients in the relation can be chosen in $Z[[\Omega, \%]]$.

\section{§3. Theta functions and abelian functions with coefficients in 2 .}

3. 1. In the following we denote by $q$ the positive symmetric bimultiplicative function on $\mathfrak{M}_{\mathrm{Q}} \times \mathfrak{M}_{\mathrm{Q}}$ valued in $\Omega^{\times}$defined in $\S 1$.

All the results in the previous section are valid for the replacement of the symbol $\mathfrak{Q}$ with $q$, if we prove the convergence of the coefficients of the products of two theta functions $\vartheta_{\alpha}[g, 0](q \mid \chi u)$ and $\vartheta_{\beta}\left[g^{\prime}, 0\right]\left(q \mid \chi^{\prime} u\right)$.

By the definition of $\mathfrak{A}\left(S^{-1}, q\right)$ we know that $\mathfrak{A}\left(S^{-1}, q\right) \otimes_{\mathrm{z}} \mathrm{Q}$ has the involu tion $*: \alpha^{*}=S^{t} \alpha S^{-1}$ and by the positive definiteness of $S$ we have $\alpha^{*} \alpha>0$ for 
$\alpha \neq 0$ in $\mathfrak{A}\left(S^{-1}, q\right)$.

LeMma 1. If $\alpha$ is a positive symmetric element in $\mathscr{H}\left(S^{-1}, q\right)$, then $v(q(\mathrm{~m}$, $\alpha \mathrm{m}))>0$ for $m \neq 0$.

Proof. Since $\alpha$ is positive symmetric, there exist a positive integer $c$ and $\beta_{1}, \ldots, \beta_{h}$ in $\mathfrak{A}\left(S^{-1}, q\right)$ such that $c \alpha=\sum_{i=1}^{h} \beta_{i}{ }^{*} \beta_{i}{ }^{1)} \quad$ From this follows $c v(q(\mathrm{~m}$, $\alpha \mathfrak{m}))=v(q(\mathfrak{m}, c \alpha \mathfrak{m}))=v\left(q\left(\mathfrak{m},\left(\sum_{i=1}^{h} \beta_{i}^{*} \beta_{i}\right) \mathfrak{m}\right)=v\left(\prod_{i=1}^{h} q\left(\mathfrak{m}, \beta_{i}^{*} \beta_{i} \mathfrak{m}\right)\right)=\sum_{i=1}^{h} v\left(q\left(\beta_{i} \mathfrak{m}\right.\right.\right.$, $\left.\left.\beta_{i} \mathfrak{m}\right)\right)$. On the other hand $\left(v\left(q\left(\mathfrak{m}_{i}, \mathfrak{m}_{j}\right)\right)\right)$ is positive definite, hence $v(q(\mathfrak{m}, \mathfrak{m}))$ $>0$ for $\mathrm{m} \neq 0$. Therefore $v(q(\mathrm{~m}, \alpha \mathrm{m}))=c^{-1} \sum_{i=1}^{h} v\left(q\left(\beta_{i} \mathfrak{m}, \beta_{i} \mathrm{~m}\right)\right)>0$ for $\mathrm{m} \neq 0$.

Lemma 2. Let $\varphi(u)$ be a theta function of type $(\alpha, \chi)$ with coefficients in $\Omega$ ( $\alpha$ is a positive symmetric element in $\left.\mathfrak{A}\left(S^{-1}, q\right)\right)$. Then $\%$ has values in $\Omega^{\times}$ on $\mathfrak{M}_{\mathrm{Q}}$. have

Proof. Putting $\varphi(u)=\sum_{\mathfrak{m} \in S^{-1} \mathfrak{M}} c_{\mathfrak{m}} u(\mathfrak{m})$ with $c_{\mathfrak{m}}$ in $\Omega$, by virtue of (17), we

$$
\begin{aligned}
\chi(\alpha \mathfrak{m}) & =\chi(\alpha(\mathfrak{m}+g)) \chi(\alpha g)^{-1} \\
& =q(\mathfrak{m}+\mathfrak{g}, \alpha(\mathfrak{m}+g))^{-1} q(g, \alpha g) c_{\alpha(\mathfrak{m}+g)} c_{\alpha g}^{-1}, \quad\left(\alpha g, \quad \mathfrak{m} \in S^{-1} \mathfrak{M}\right) .
\end{aligned}
$$

This shows that $\chi(\alpha m)\left(m \in S^{-1} \mathfrak{M}\right)$ belong to $\Omega^{\times}$. On the other hand $\alpha$ is positive, hence for any $n$ in $\mathfrak{M}_{\mathrm{Q}}$ there exists a positive integer $\nu$ such that $\nu \mathfrak{n} \in S^{-1} \mathfrak{M}$; Namely $\chi(\mathfrak{n})^{\nu}=\chi(\nu \mathfrak{n})$ belongs to $\Omega^{\times}$. Since $\Omega$ is algebraically closed, $\chi(\mathfrak{n})$ belongs to $\Omega$.

PROPOSITION 8. Let $\chi, \chi^{\prime}$ be multiplicative functions on $\mathfrak{M}_{\mathrm{Q}}$ valued in $\Omega^{\times}$ and $\alpha, \beta$ be positive symmetric elements in $\mathfrak{A}\left(S^{-1}, q\right)$. Then the series: $\sum_{\mathfrak{n} \in S^{-1} \mathfrak{M}} q(\mathfrak{n}+g, \alpha(\mathfrak{n}+\mathfrak{g})) q\left(\beta^{-1}(\mathfrak{m}-\alpha(\mathfrak{n}+g)), m-\alpha(\mathfrak{n}+g)\right) \chi(\alpha(\mathfrak{n}+g)) \chi^{\prime}(\mathfrak{m}-\alpha(\mathfrak{n}$ $+g)$ ) converges for every $\mathrm{g}, \mathfrak{m}$ in $\mathfrak{M}_{Q}$.

Proof. We shall denote by $I(\mathfrak{n})$ the $\mathfrak{n}$-term

$$
q(\mathfrak{n}+\mathfrak{g}, \alpha(\mathfrak{n}+\mathfrak{g})) q\left(\beta^{-1}(\mathfrak{m}-\alpha(\mathfrak{n}+\mathfrak{g})), \mathfrak{m}-\alpha(\mathfrak{n}+\mathfrak{g})\right) \chi(\alpha(\mathfrak{n}+\mathfrak{g})) \chi^{\prime}(\mathfrak{m}-\alpha(\mathfrak{n}+\mathfrak{g})) .
$$

Since it is sufficient to prove for non-archimedean case, it is sufficient to show that for any positive integer $N$, there exists a positive integer $M$ such that, if $\sum_{i=1}^{r}\left|x_{i}(\mathfrak{n})\right|>M$ then $v(I(\mathfrak{n}))>N$. Put $a_{i j}=v\left(q\left(\mathrm{in}_{i}, \alpha \mathrm{m}_{j}\right)\right.$ and $b_{i j}=v\left(q\left(\beta^{-1} \alpha \mathrm{m}_{i}\right.\right.$, $\left.\alpha m_{j}\right)$ ). Then, since $\alpha$ and $\beta$ are positive symmetric elements in $\mathfrak{A}\left(S^{-1}, q\right)$ by

1) See [1] p. 482 . 
virtue of Lemma 1 , the symmetric matrices $\left(a_{i j}\right)$ and $\left(b_{i j}\right)$ are positive definite. The principal part (as a polynomial in $\left.x_{1}(\mathfrak{n}), \ldots, x_{r}(\mathfrak{n})\right)$ of $v\left(I(\mathfrak{n})\right.$ is $\sum\left(a_{i j}\right.$ $\left.+b_{i j}\right) x_{i}(\mathfrak{n}) x_{j}(\mathfrak{n})$, hence, by the positive definiteness of the matrix $\left(a_{i j}+b_{i j}\right)$ we see that for any positive integer $N$ there exists a positive integer $M$ such that if $\sum_{i=1}^{r}\left|x_{i}(\mathfrak{n})\right|>M$, then $v(I(\mathfrak{n}))>N$.

Proposition 9. $\vartheta_{\alpha}\left[g_{i}, 0\right](q \mid u) \quad(1 \leq i \leq \operatorname{det} \alpha)$ converges for every multiplicative function $\chi$ on $M_{\mathrm{Q}}$ valued in $\Omega^{\times}$.

Proof. In the complex case, this is classically known, so it is sufficient to prove for non-archimedean $v$. Let us culculate the value:

$$
\begin{aligned}
v(q(\mathrm{~m} & +g, a(\mathrm{~m}+\mathrm{g})) \chi(\alpha(\mathrm{m}+\mathrm{g})))=\sum_{i, j=1}^{r} x_{i}(\mathrm{~m}) x_{j}(\mathrm{~m}) v\left(q\left(\mathrm{~m}_{i}, \alpha \mathrm{m}_{j}\right)\right) \\
& +\sum_{i=1}^{r} x_{i}(\mathrm{~m})\left(v\left(q\left(\mathrm{~m}_{i}, \alpha g\right)+v\left(q\left(g, \alpha \mathrm{m}_{i}\right)\right)+v\left(\chi\left(\alpha \mathrm{m}_{i}\right)\right)\right)\right. \\
& +v(q(g, \alpha g))+v(\mathcal{g}(\alpha g)) .
\end{aligned}
$$

By virtue of Lemma $1,\left(v\left(q\left(\mathfrak{m}_{i}, \alpha m_{i}\right)\right)\right)$ is positive definite, hence, for any positive integer $N$, there exists a positive integer $M$ such that $v(q(m+g, \alpha(m$ $+g))>N$ for every $m$ in $S^{-1} \mathfrak{M}$ satisfying $\sum_{i=1}^{r}\left|x_{i}(m)\right|>M$. This proves the convergence of $\vartheta_{\alpha}[\mathrm{g}, 0](q \mid u)$.

By Proposition 8, Lemma 2 and (18), we have

Proposition 10. Let $\varphi(u)$ be a theta function of type $(\alpha, \%)$ with coefficients in $\Omega$ (with the period $\left(S^{-1}, q\right)$ ). Then, $\varphi(\xi)$ converges for every multiplicative function $\xi$ valued in $\Omega^{\times}$.

3.2. By virtue of Proposition 4, the product of abelian functions with coefficients in $\Omega$ and with period $\left(S^{-1}, q\right)$ is also an abelian function of the same type. Obviously the differences, the sums and the inverses of abelian functions are also abelian functions. Hence all the abelian functions with coefficients in $\Omega$ and with period $\left(S^{-1}, q\right)$ form a field $K_{S, q}$ over $\Omega$. We shall now prove the following theorem:

ThEOREM $1 . K_{S, q}$ is a finitely and separably generated extension of dimension $r$ over $\Omega$.

Proof. Putting $F_{n i}(u)=\vartheta_{n}\left[n^{-1} \mathfrak{m}_{i}, 0\right](q \mid u) / \vartheta_{n}[0,0](q \mid u)(n>1 ; 1 \leq i \leq r)$, we shall prove that (if $p \neq 0) K_{s, q} / \Omega\left(F_{p 1}(u), \ldots, F_{p r}(u)\right)$ is a finite separable 
algebraic extension and $K_{s, q} / \Omega\left(F_{n_{1}}(u), \ldots, F_{n r}(u)\right)$ is a finite algebraic extension. It is sufficient to prove the former assertion, because, by virtue of Proposition $5, F_{n 1}(u), \ldots, F_{n r}(u)$ are algebraically independent over $\Omega$. First assume, for a moment, $K_{s, q}$ is not separable over $\Omega\left(F_{p 1}(u), \ldots, F_{p r}(u)\right)$. Then there exist an element $f$ in $K_{s, q}$ and an irreducible polynomial $\sum_{i=0}^{n} a_{i} X^{i}$ with coefficients in $\Omega\left(F_{p 1}, \ldots, F_{p r}\right)$ such that $\sum_{i=0}^{n} a_{i} f^{p i}=0$. Let $\varphi$ and $\psi$ be theta functions such that $f=\varphi / \psi$. Then the relation induces a homogeneous relation of $\varphi, \psi, \vartheta_{p}[0,0], \vartheta_{p}\left[p^{-1} \mathfrak{m}_{i}, 0\right](1 \leq i \leq r)$ with coefficients in $\Omega$ :

(*) $\quad \sum_{i=0}^{n} b_{l ; i_{0} \cdots i_{r}} \vartheta_{p}[0,0]^{i_{0}} \cdots \vartheta_{p}\left[p^{-1} m_{r}, 0\right]^{i_{r}} \varphi^{l p} \psi^{(n-l) p}=0$,

where we may assume that the degree $d=\sum_{l=0}^{r} i_{l}$ on $\vartheta_{p}[0,0], \vartheta_{p}\left[p^{-1} \mathfrak{m}_{1}, 0\right]$, $\ldots, \vartheta_{p}\left[p^{-1} m_{r}, 0\right]$ in the relation is the lowest of the possible cases and the number of terms is also the least value provided that the degree on $\vartheta$ is $d$. Since the degree of $u_{j}$ in each term of $\vartheta_{p}\left[p^{-1} m_{i}, 0\right]$ is $\delta_{i j}$ modulo $p(1 \leq i, j \leq r)$ and the degree $u_{j}$ in each term $\vartheta_{p}[0,0], \varphi^{l p}, \psi^{(n-l) p}$ is zero modulo $p$. This shows the degree of $u_{j}$ in each term of $\vartheta_{p}[0,0]^{i_{0}} \vartheta_{p}\left[p^{-1} \mathrm{~m}_{1}, 0\right]^{i_{1}} \cdots \vartheta_{p}\left[p^{-1} \mathrm{~m}_{r}\right.$, $0]^{i r} \varphi^{l p} \psi^{(n-l) p}$ is $i_{j}$ modulo $p$. This means that the degree of $\vartheta_{p}\left[p^{-1} \mathrm{~m}_{i}, 0\right]$ in each term of $(*)$ is congruent to the same value, say $\lambda_{j}$, modulo $p$, for otherwise

$$
\sum_{\substack{j_{1} \equiv i_{1} \\ \vdots \\ j_{r} \approx i_{r}}} b_{l ; j_{0} \cdots j_{r}} \vartheta_{p}[0,0]^{j_{0}} \cdots \vartheta_{p}\left[p^{-1} \mathrm{~m}_{r}, 0\right]^{j_{r}} \varphi^{l_{p}} \psi^{(n-l i p}=0
$$

is a shorter relation than $(*)$. If $\lambda_{j} \neq 0 \bmod p$, we can get a lower relation. So $\lambda_{j} \equiv 0 \bmod p$ and consequently all degree $i_{0}$ of $\vartheta_{p}[0,0]$ in $(*)$ are also congruent to the same value, say $\lambda_{0}$, modulo $p$. Let $h$ be the integer $0 \leq h<p$, such that $h+\lambda_{0} \equiv 0 \bmod p$. Then, putting $\mu_{0}=p^{-1}\left(i_{0}+h\right), \mu_{j}=p^{-1} i_{j}, a_{l ; \mu_{0} \cdots \mu_{r}}=b_{l ; i_{0} \cdots i_{r}}^{p-1}$, we have $\sum_{l=1}^{n} \sum_{(\mu)} a_{l ; \mu_{0} \ldots \mu_{r}} \vartheta_{p}[0,0]^{\mu_{0}} \vartheta_{p}\left[p^{-1} \mathrm{~m}_{1}, 0\right]^{\mu_{1}} \cdots \vartheta_{p}\left[p^{-1} \mathrm{~m}_{r}, 0\right]^{\mu_{r}} \varphi^{l} \psi^{(n-l) p}=0$. This shows $f=\varphi / \psi$ is separable over $\Omega\left(F_{p_{1}}, \ldots, F_{p r}\right)$. This is a contradiction. Namely $K_{s, q} / \Omega\left(F_{p 1}, \ldots, F_{p r}\right)$ is separable. On the other hand, by virtue of Proposition 6, every element $f$ in $K_{S, q}$ is of bounded degree over $\Omega\left(F_{p_{1}}, \ldots\right.$, $\left.F_{p r}\right)(p \neq 0)$, hence $K_{S, q} / \Omega\left(F_{p 1}, \ldots, F_{p r}\right)$ is an algebraic simple extension of finite degree. Since by virtue of Corollary of Proposition $6, \Omega\left(F_{n_{1}}, \ldots, F_{n r}\right.$, $\left.F_{p 1}, \ldots, F_{p r}\right) / \Omega\left(F_{n 1}, \ldots, F_{n r}\right)$ is a finite algebraic extension, $K_{s, q} / \Omega\left(F_{n 1}\right.$, $\left.\ldots, F_{n 1}\right)$ is also a finite algebraic extension. Since every element in $K_{S, q}$ is 
a bounded degree over $\Omega\left(F_{n 1}, \ldots, F_{n r}\right)$, if $p \neq 0, K_{s, q}$ is a finite algebraic extension of $\Omega\left(F_{n 1}, \ldots, F_{n r}\right)$. This completes the proof of Theorem 1 .

3. 3. We shall now prove the Addition Theorem of abelian functions with coefficients in $\Omega$.

Lemma 3. Let $f(u)$ be a non-zero abelian function in $K_{s, 4}$. Then there exists $\xi$ in $(\xi(\Omega)$ such that $f(\xi) \neq 0$.

Proof. By virtue of the definition of abelian function there exist theta functions $\varphi$ and $\psi$ of some type $(n, \%)$ with coefficients in $\Omega$ such that $f=\varphi / \psi$. Since $\varphi$ and $\phi$ are power series in $u_{1}, \ldots, u_{r}, u_{1}^{-1}, \ldots, u_{r}^{-1}$, we may consider them as analytic functions on $\Omega^{\times} \times \overbrace{\cdots \times} \Omega^{\times}$. So the quotient $f$ is not always zero on $\Omega^{\times} \times \cdots \times \Omega^{\times}$.

We call an element $w$ in $(\xi(\mathrm{K})$ to be variable over a subfield $L$ in $\mathrm{K}$ if there exists no non-trivial relations $\sum_{\mathfrak{M} \in n^{-1} \mathfrak{M}} c_{\mathfrak{m}} w(\mathfrak{m})=0$ with coefficients $c_{\mathfrak{m}}(\mathrm{m}$ $\left.\in n^{-1} \mathfrak{M}\right)$ in $L$. Then simalarly as Lemma 3 we have.

Lemma $3^{\prime}$. Let $w$ be a multiplicative function on $\mathfrak{M}_{\mathrm{Q}}$ variable over $\Omega$ and let $f(u)$ be a non-zero abelian function in $\Omega((w)) K_{s, q}$. Then there exists a multiplicative function $\xi$ in $(\xi(\Omega)$ such that $f(\xi) \neq 0$.

Lemma 4. Let $w$ be a multiplicative function on $\mathfrak{M}_{\mathrm{Q}}$ variable over $\Omega$. Let $f_{1}, \ldots, f_{N}$ be abelian functions in $K_{S, q}$ (resp. $\left.\Omega((w)) K_{s, q}\right)$ linearly independent over $\Omega$ (resp. $\Omega((w)))$. Then there exist multiplicative functions $\xi_{1}, \ldots, \xi_{N}$ in $(5)$ such that

$$
\operatorname{det}\left(f_{i}\left(\xi_{j}\right)\right) \neq 0 .
$$

Proof. We shall prove the Lemma by the induction on $N$. By virtue of Lemma 3 (resp. Lemma $3^{\prime}$ ), for $N=1$ the Lemma is true. If we assume the Lemma for $N-1$ we have multiplicative functions $\xi_{1}, \ldots, \xi_{. v-1}$ in $\leftleftarrows(\Omega)$ such that

$$
\operatorname{det}\left(\begin{array}{c}
f_{1}\left(\xi_{1}\right), \ldots, f_{1}\left(\xi_{N-1}\right) \\
f_{N-1}\left(\xi_{1}\right), \ldots, f_{N-1}\left(\xi_{N-1}\right)
\end{array}\right) \neq 0 .
$$

Since $f_{1}, \ldots, f_{N}$ are linearly independent over $\Omega$ (resp. $\Omega((w))$, we have

$$
\operatorname{det}\left(\begin{array}{c}
f_{1}\left(\xi_{1}\right), \ldots, f_{1}\left(\xi_{N-1}\right), f_{1}(u) \\
f_{N}\left(\xi_{1}\right), \ldots, f_{N}\left(\xi_{N-1}\right), f_{N}(u)
\end{array}\right) \neq 0 .
$$


Hence, from Lemma 3 (resp. Lemma $3^{\prime}$ ), there exists a multiplicative function $\xi_{N}$ in $\xi(\Omega)$ such that $\xi_{1}, \ldots, \xi_{N}$ satisfying the condition of the Lemma.

LemMa 5. Let $\varphi$ and $\psi$ be theta functions of type $\left(n, \chi_{0}\right)$ with coefficients in $\Omega$ and $\chi$ be a multiplicative function on $\mathbb{M}_{Q}$. Put $f(u)=\varphi(\mathcal{\chi} u) / \psi(\chi u)$. Then $f$ belongs to $\Omega((\chi)) K_{s, q}$.

Proof. It is sufficient to prove for variable $\chi$. We shall denote by $K_{0}$ the field $\Omega\left(F_{p 1}, \ldots, F_{p r}\right)$ for $p \neq 0$ and $\Omega\left(F_{n 1}, \ldots, F_{n r}\right)$ for $p=0$, where $F_{p i}$, $F_{n j}(1 \leq i, j \leq r)$ are the quotients of the theta functions in Theorem 1 . Then, by virtue of Theorem $1, K_{s, q} / K_{0}$ is a separable extension of finite degree. Assume $\Omega((\mathcal{\chi})) K_{S, q}$ does not contain $f$ and denote by $\nu$ the degree of $f$ over $\Omega((\chi)) K_{s, q} . \quad$ Let $\chi_{1}, \ldots, \chi_{t}$ be independent generic specializations of $\chi$ over $\Omega$ and $f_{1}, \ldots, f_{t}$ be the images of $f$ by the specializations. First we shall prove that $\left\{f_{1}^{i_{1}} \cdots f_{t}^{i_{t}} \mid 1 \leq i_{l} \leq \nu-1 ; 1 \leq l \leq r\right\}$ are independent over $\Omega\left(\left(\chi_{1}\right.\right.$, $\left.\left.\ldots, \chi_{t}\right)\right) K_{s, q}$. Assume there exists a non-trivial relation

(*) $\sum_{\substack{0 \leq i_{1} \leq \nu-1 \\ \vdots \\ 0 \leq i_{r} \leq \nu-1}} a_{i_{1}} \ldots i_{t}\left(\chi_{1}, \ldots, \chi_{t}\right) f_{1}^{i_{1}} \cdots f_{t}^{i_{t}}=0$

with coefficients in $\left.\Omega\left(\%_{1}, \ldots, \psi_{t}\right)\right) K_{s, q}$, where we may assume $(*)$ is nontrivial on $f$. Specializing $\chi_{2}, \ldots, \chi_{t}$ to suitable multiplicative functions on $\mathfrak{M}_{\mathbf{Q}}$ valued in $\Omega^{\times}$such that the specialized relation of $(*)$ is non-trivial on $f$, we have a contradiction with $\left[\Omega((\chi)) K_{s, q}(f): \Omega((\chi)) K_{s, q}\right]=\nu$. This proves

$$
\left[\Omega\left(\left(\chi_{1}, \ldots, \chi_{t}\right)\right) K_{s, q}\left(f_{1}, \ldots, f_{t}\right): \Omega\left(\left(\chi_{1}, \ldots, \chi_{t}\right)\right) K_{s, q}\right]=\nu^{t} .
$$

This contradicts Proposition 6 for sufficiently large $t$.

Theorem 2. (The Addition Theorem). Let $f_{1}, \ldots, f_{N}$ be generators of $K_{s, q}$ over $\Omega$ and $f$ be any abelian function in $K_{s, q}$. Then $f(u w)$ belongs to $\Omega\left(f_{1}(u), \ldots, f_{N}(u), f_{1}(w), \ldots, f_{N}(w)\right)$ and $f\left(u^{-1}\right)$ belongs to $\Omega\left(f_{1}(u), \ldots\right.$ $\left.f_{N}(u)\right)$.

Proof. Let $w$ be a multiplicative function on $\mathfrak{M}_{\mathrm{Q}}$ and put $g_{w}(u)=f(w u)$. Then, by virtue of Lemma $5, g_{w}(u)$ belongs to $\Omega((w)) K_{s, q}$. Therefore there exist abelian functions $\varphi_{1}, \ldots, \varphi_{M}$ in $K_{S, q}$ linearlly independent over $\left.\Omega((w))\right)$ and elements $b_{1}(w), \ldots, b_{M}(w), c_{1}(w), \ldots, c_{M}(w)$ in $\Omega((w))$ such that

(*) $\quad g_{w}(u)=f(w u)=\sum_{i=\mathrm{f}}^{M} b_{i}(w) \varphi_{i}(u) / \sum_{i=1}^{M} \mathrm{c}_{i}(w) \varphi_{i}(u)$. 
We may assume that the number $M$ of $\left\{\varphi_{i}(\boldsymbol{u})\right\}$ is the least value of possible cases and, after changing the indices, we may assume $c_{\mathbb{M}}(w)=1$. From Lemma 4 , there exist multiplicative functions $\xi_{1}, \ldots, \xi_{M}$ on $\mathfrak{M}_{Q}$ valued in $\Omega^{\times}$such that $\operatorname{det}\left(\varphi_{i}\left(\xi_{j}\right)\right) \neq 0$. After translating $\varphi_{1}, \ldots, \varphi_{M}$ by the matrix $\left(\varphi_{i}\left(\xi_{j}\right)\right)$, we may assume that $\left(\varphi_{i}\left(\xi_{j}\right)\right)$ is the unit matrix. Hence by $(*)$, we have

$$
\begin{aligned}
& b_{i}(w)=f\left(\xi_{i} w\right) c_{i}(w) \\
& b_{M}(w)=f\left(\xi_{M} w\right),
\end{aligned}
$$

and

$(* *) \quad \sum_{i=1}^{M-1}\left\{\left(f(w u)-f\left(\xi_{i} w\right)\right) \varphi_{i}(u) c_{i}(w)\right\}+\left(f(w u)-f\left(\xi_{M} w\right)\right) \varphi_{M}(u)=0$.

$\left\{\left(f(w u)-f\left(\xi_{i} w\right)\right) \varphi_{i}(u),(1 \leq i \leq M-1)\right\}$ are linearly independent over $\Omega$, for, otherwise $\sum_{i=1}^{M-1} d_{i}\left(f(w u)-f\left(\xi_{i} w\right)\right) \varphi_{i}(u)=0, \quad f(w u)=\sum_{i=1}^{M-1} d_{i} f\left(\xi_{i} w\right) \varphi_{i}(u) / \sum_{i=1}^{M-1} d_{i} \varphi_{i}(u)$ and this contradicts the assumption on $M$. Therefore by virtue of Lemma 4 , we have $M-1$ multiplicative functions $\eta_{1}, \ldots, \eta_{u-1}$ on $\mathfrak{M}_{\mathbf{Q}}$ valued in $\Omega^{\times}$such that $\operatorname{det}\left(\psi_{i}\left(\eta_{j}\right)\right) \neq 0$, where $\left.\psi_{i}(u)=(f(w u))-f\left(\xi_{i} w\right)\right) \varphi_{i}(u)$. Hence, from $(* *)$, $c_{i}(w)(1 \leq i \leq M-1)$ are rational function of $\left(f\left(w \eta_{j}\right)-f\left(\xi_{i} w\right)\right) \varphi_{i}\left(\eta_{j}\right)(1 \leq i \leq M$; $1 \leq j \leq M-1)$. This shows that $b_{i}(w), c_{j}(w)(1 \leq i \leq M ; 1 \leq j \leq M-1)$ are abelian functions in $\Omega\left(f_{1}(w), \ldots, f_{N}(w)\right)$, because $f\left(w \eta_{i}\right), f\left(\xi_{j} w\right) \in \Omega\left(f_{1}(w)\right.$, $\left.\ldots, f_{V}(w)\right)(1 \leq i, j \leq M)$. This shows $f(w u) \in Q\left(f_{1}(u), \ldots, f_{N}(u), f_{1}(w)\right.$, $\left.\ldots, f_{N}(w)\right)$. By virtue of (18) any theta function of type $(n, \chi)$ is a linear combination of $\vartheta_{n}[g, 0](q \mid u \chi)\left(n g \in S^{-1} \mathfrak{M}\right)$ and $\vartheta_{n}[g, 0](q \mid u)=\vartheta_{n}[-g, 0]\left(q \mid u^{-1}\right)$. This means that, if $\varphi(u) / \psi(u)$ is the quotient of theta functions $\varphi, \psi$ of type $(n, \chi)$ with coefficients in $\Omega, \varphi\left(u^{-1}\right) / \psi\left(u^{-1}\right)$ is also the quotient of theta functions $\varphi^{*}(u)=\varphi\left(u^{-1}\right), \psi^{*}(u)=\psi\left(u^{-1}\right)$ with coefficients in $\Omega$. This proves the last assertion of the Theorem.

3.4. We shall prove the multiplicative theorem.

Proposition 11. Let $\alpha$ be a positive symmetric element in $\mathfrak{A}\left(S^{-1}, q\right), \beta$ be a non-singular element in $\mathfrak{H}\left(S^{-1}, q\right)$ and $\varphi$ be a theta function of type $(\alpha, \chi)$ with coefficients in $\Omega$. Put $\phi(u)=\varphi\left(u^{\beta}\right)\left(u^{\beta}(m)\right.$ means $\left.u(\beta m)\right)$. Then $\psi(u)$ is $a$ theta function of type $\left(\beta \alpha \beta^{*}, \%\right)$ with coefficients in $\Omega$.

Proof. From the definition of theta functions of type $(\alpha, \%)$ and multiplications of $\left(S^{-1}, q\right)$, for any $m$ in $S^{-1} \mathfrak{M}$ we have 


$$
\begin{aligned}
\psi(g(\mathrm{~m}) u) & =\varphi\left(g(\mathrm{~m})^{\beta} u^{\beta}\right)=\varphi\left(q\left(\beta^{*} \mathrm{~m}\right) u^{\beta}\right) \\
& =q\left(\beta^{*} \mathrm{~m}, \alpha \beta \mathrm{m}\right)^{-1} u\left(\beta \alpha \beta^{*} \mathrm{~m}\right)^{-1} \chi\left(\beta \alpha \beta^{*} \mathrm{~m}\right)^{-1} \varphi(u \beta) \\
& =q\left(\mathrm{~m}, \beta \alpha \beta^{*} \mathrm{~m}\right)^{-1} u\left(\beta \alpha \beta^{*} \mathrm{~m}\right)^{-1} \chi\left(\beta \alpha \beta^{*} \mathrm{~m}\right) \varphi(u) .
\end{aligned}
$$

Proposition 12. Let $\varphi_{1}$ and $\varphi_{2}$ be theta functions of type $(\alpha, \%)$ with coefficients in $\Omega$. Then there exist a positive integer $n$ and theta functions $\psi_{1}$ and $\psi_{2}$ of type $(n, \gamma)$ with coefflcients in $\Omega$ such that

$$
\varphi_{1}(\boldsymbol{u}) / \varphi_{2}(\boldsymbol{u})=\psi_{1}(\boldsymbol{u}) / \psi_{2}(\boldsymbol{u}) .
$$

Proof. Let $n$ be a positive integer such that $\beta=n-\alpha$ is a positive symmetric element in $\mathfrak{H}\left(S^{-1}, q\right)$. Put

$$
\begin{aligned}
& \psi_{1}(u)=\varphi_{1}(u) \vartheta_{\beta}[0,0](q \mid \chi u) \text { and } \\
& \psi_{2}(u)=\varphi_{2}(u) \vartheta_{\beta}[0,0]\left(q \mid \chi_{u}\right) .
\end{aligned}
$$

Then $\psi_{1}$ and $\psi_{2}$ satisfies the condition in the Proposition.

By virtue of Proposition 12, abelian functions in $K_{s, q}$ are defined by the quotients of theta functions of the same types $(\alpha, \chi)$ with coefficients in $\Omega$, where $\alpha$ runs over positive symmetric elements of $\mathfrak{A}\left(S^{-1}, q\right)$.

TheOREM 3. (The Multiplication Theorem). Let $\alpha$ be a multiplication of $\left(S^{-1}, q\right)$ and $f$ be an abelian function in $K_{s, q}$. Put $g(u)=f\left(u^{\alpha}\right)$. Then $g$ belongs to $K_{s, a}$.

Proof. Let $\varphi_{1}$ and $\varphi_{2}$ be theta functions of type $(n, \chi)(n \in Z)$ with coefficients in $\Omega$ such that $f(u)=\varphi_{1}(u) / \varphi_{2}(u)$. Put $\phi_{1}(u)=\varphi_{1}\left(u^{\alpha}\right)$ and $\phi_{2}(u)$ $=\varphi_{2}\left(u^{\alpha}\right)$. Then, by virtue of Proposition $11, \psi_{1}(u)$ and $\psi_{2}(u)$ are theta functions of type ( $\left.n \alpha \alpha^{*}, \chi\right)$ with coefficients in $\Omega$ such that $g(u)=\phi_{1}(u) / \psi_{2}(u)$. Therefore, from Proposition 12, we have $g \in K_{s, q}$.

\section{§4. Abelian variety with period $\left(S^{-1}, q\right)$}

4.1. By virtue of the addition theorem of abelian functions in $K_{s, q}$, we can now prove that $K_{S, q}$ is canonically isomorphic to the field of rational functions on an abelian variety $A_{s, q}$ and the law of composition on $A_{s, q}$ is induced by the multiplication $(v, w) \rightarrow v w$ of multiplicative functions $v, w$ on $\mathfrak{M}_{\mathrm{Q}}$.

We shall mean by $\mathbb{E}\left(S^{-1}, q\right)$ the subgroup $\left\{q(\mathrm{~m}) \mid \mathrm{m} \in S^{-1} \mathfrak{M}\right\}$ of $\mathbb{E}(\mathrm{K})$ and $\mathfrak{I}_{S, q}$ the quotient group of $\mathbb{E}(\mathrm{K})$ by $\mathbb{E}\left(S^{-1}, q\right)$. We shall mean by $\mathfrak{I}_{S, q}(\Omega)$ the quotient group $\&(\Omega) / \mathbb{E}\left(S^{-1}, q\right)$. 
Lemma 6. Let $\mathfrak{g}, \mathfrak{h}$ be elements in $n^{-1} S^{-1} \mathfrak{M}$ such that $\mathfrak{g} \notin S^{-1} \mathfrak{M}$. Then

$$
\vartheta_{n}[-\mathfrak{g}, 0](q \mid q(\mathfrak{g}) 1(\mathfrak{h})) \neq 0
$$

and

$$
\begin{aligned}
\vartheta_{n}[-\mathfrak{g}, 0](q \mid g(g) 1(h)) & : \vartheta_{n}[0,0](q \mid q(\mathfrak{g}) 1(\mathfrak{h})) \\
& \neq \vartheta_{n}[-g, 0](q \mid 1): \vartheta_{n}[0,0](q \mid 1) .
\end{aligned}
$$

Proof. By the periodical properties of $\vartheta$ we have

$$
\begin{aligned}
\vartheta_{n}[-\mathfrak{g}, 0](q \mid q(\mathfrak{g}) 1(\mathfrak{h})) & =\sum_{\mathfrak{m} \in S^{-1} \mathfrak{M}} q(\mathfrak{m}-g, n(\mathfrak{m}-\mathfrak{g})) 1(\mathfrak{h}, n(\mathfrak{l}-g)) q(g, n(\mathfrak{m}-\mathfrak{g})) \\
& =q(\mathfrak{g}, n \mathfrak{g})^{-1} 1(\mathfrak{G}, n \mathfrak{g}) \sum_{\mathfrak{m} \in S^{-1} \mathfrak{M}} q(\mathfrak{m}, n \mathfrak{m}) \\
& =q(g, n g)^{-1} 1(\mathfrak{h}, n \mathfrak{g}) \vartheta_{n}[0,0](q \mid 1)
\end{aligned}
$$

and

$$
\begin{aligned}
\vartheta_{n}[0,0](q \mid q(\mathfrak{g}) 1(\mathfrak{G})) & =\sum_{\mathfrak{m} \in S^{-1} \mathfrak{M}} q(\mathfrak{m}, n \mathrm{~m}) 1(\mathfrak{h}, n \mathfrak{m}) q(\mathfrak{g}, n \mathfrak{m})^{2} \\
& =q(\mathfrak{g}, n \mathfrak{g})^{-1} \sum_{\mathfrak{m} \in S^{-1} \mathfrak{M}} q(\mathfrak{m}+g, n(\mathfrak{m}+g)) \\
& =q(g, n g)^{-1} \vartheta_{n}[\mathfrak{g}, 0](q \mid 1) .
\end{aligned}
$$

Since $\vartheta_{n}[-g, 0](q \mid 1)=\vartheta_{n}[g, 0](q \mid 1)$, we have

$$
\vartheta_{n}[0,0](q \mid g(\mathfrak{g}) \mathcal{I}(\mathfrak{G}))=q(g, n g)^{-1} \vartheta_{n}[-g, 0](q \mid 1) .
$$

On the other hand, since $v(q(m, n m))>0$ for $m \neq 0$, we have

$$
v\left(\vartheta_{n}[0,0](q \mid 1)\right)=v(1)=0
$$

and

$$
v\left(\vartheta_{n}[-g, 0](q \mid 1)\right)>\operatorname{Min} v(q(m-g, n(m-g)))>0 .
$$

This implies

$$
v\left(\vartheta_{n}[-g, 0](q \mid 1) / \vartheta_{n}[0,0](q \mid 1)\right)>0
$$

and

$$
\begin{aligned}
& v\left(\vartheta_{n}[-g, 0](q \mid q(\mathfrak{g}) 1(\mathfrak{h}))\right) \\
& \quad=v\left(g(g, n g)^{-1} 1(\mathfrak{h}, n g) \vartheta_{n}[0,0](q \mid 1)\right) \\
& =v\left(q(g, n g)^{-1}\right)<0 .
\end{aligned}
$$

Hence

This shows

$$
\begin{aligned}
v\left(\vartheta_{n}[-g, 0](q \mid q(\mathfrak{g}) 1(\mathfrak{h})) / \vartheta_{n}[0,0](q \mid q(\mathfrak{g}) 1(\mathfrak{h}))\right) \\
=v\left(1(\mathfrak{h}, n \mathfrak{g}) \vartheta_{n}[0,0](q \mid 1) / \vartheta_{n}[-\mathfrak{g}, 0](q \mid 1)\right) \\
=v\left(\vartheta_{n}[0,0](q \mid 1) / \vartheta_{n}[-\mathfrak{g}, 0](q \mid 1)\right)<0
\end{aligned}
$$




$$
\vartheta_{n}[-g, 0](q \mid q(g) 1(h)) \neq 0
$$

and

$$
\begin{aligned}
\vartheta_{n}[-g, 0](q \mid q(g) 1(\mathfrak{h})) & : \vartheta_{n}[0,0](q \mid q(g) 1(\mathfrak{h})) \\
& \neq \vartheta_{n}[-g, 0](q \mid 1): \vartheta_{n}[0,0](q \mid 1) .
\end{aligned}
$$

Lемма 7. Let $l$ be a prime number different from $p$ and $g$ be an element in $l^{-1} S^{-1} \mathfrak{M}$ such that $\mathfrak{g} \notin S^{-1} \mathfrak{M}$. Then there exist a positive integer $\nu$ and an element $\mathfrak{h}$ in $l^{-\nu} S^{-1} \mathfrak{M}$ such that

$$
\vartheta_{l}[\mathfrak{h}, 0](q \mid 1(\mathfrak{g})) \neq 0
$$

and

$$
\begin{aligned}
\vartheta_{l v}[\mathfrak{h}, 0](q \mid 1(\mathfrak{g})): & \vartheta_{l \nu}[0,0](q \mid 1(\mathfrak{g})) \\
& \neq \vartheta_{l v}[\mathfrak{h}, 0](g \mid 1): \vartheta_{l}[0,0](q \mid 1) .
\end{aligned}
$$

Proof. Since $\left(v\left(g\left(\mathrm{~m}_{i}, \mathrm{~m}_{j}\right)\right)\right.$ is positive definite, there exists a positive real munber $\varepsilon$ such that if ${ }^{t} \mathfrak{h} S^{2} \mathfrak{h}<\varepsilon$ implies $v(q(\mathfrak{h}, \mathfrak{h}))<v(q(\mathfrak{m}+\mathfrak{h}, \mathfrak{m}+\mathfrak{h}))$ for every non-zero $\mathrm{m}$ in $S^{-1} \mathfrak{M}$. Putting $g=\sum_{i=1}^{r} x_{i}(g) m_{i}$, we assume $x_{i}(g)$ is not an $l$-integer. Let $\nu$ be a positive integer such that $l^{-2 v}<\varepsilon$. Then we have

$$
\begin{aligned}
\vartheta_{l v}\left[l^{-\nu} \mathrm{m}_{i}, 0\right](q \mid 1(g)) & =\sum_{\mathfrak{m} \in S^{-1} \mathfrak{M}} q\left(\mathrm{~m}+l^{-\nu} \mathrm{m}_{i}, l^{\nu} \mathrm{m}+\mathrm{m}_{i}\right) 1\left(\mathrm{~g}, l^{\nu} \mathrm{m}+\mathrm{m}_{i}\right) \\
& =1\left(\mathrm{~g}, \mathrm{~m}_{i}\right) \sum_{\mathfrak{m} \in S^{-1} \mathfrak{M}} q\left(\mathrm{~m}+l^{-\nu} \mathrm{m}_{i}, l^{\nu} \mathrm{m}+\mathrm{m}_{i}\right) \\
& =1\left(\mathrm{~g}, \mathrm{~m}_{i}\right) \vartheta_{l v}\left[l^{-\nu} \mathrm{m}_{i}, 0\right](q \mid 1)
\end{aligned}
$$

and

$$
\vartheta_{l \nu}[0,0](q \mid 1(g))=\sum_{m \in S^{-1} \mathfrak{M}} q\left(\mathrm{~m}, l^{\nu} \mathrm{m}\right) 1\left(g, l^{\nu} \mathrm{m}\right)=\vartheta_{l \nu}[0,0](q \mid 1) .
$$

On the other hand, $l^{-2 \nu t} \mathrm{~m}_{i} S^{2} \mathrm{~m}_{i}=l^{-2 \nu}<\varepsilon$, hence $v\left(q\left(l^{-\nu} \mathrm{m}_{i}, \mathrm{in}_{i}\right)<v\left(q\left(\mathrm{~m}+l^{\nu} \mathrm{m}_{i}\right.\right.\right.$, $\left.l^{\nu} \mathrm{m}+\mathrm{m}_{i}\right)$ ) for every non-zero $\mathrm{m}$ in $S^{-1} \mathfrak{M}$. This shows $v\left(\vartheta_{l}\left[l^{-2} \mathrm{~m}_{i}, 0\right](q \mid 1)\right)$ $=v\left(q\left(l^{-\nu} \mathrm{m}_{i}, \mathrm{~m}_{i}\right)\right.$ and $\vartheta_{l}\left[l^{-\nu} \mathrm{m}_{i}, 0\right](q \mid 1) \neq 0$. Since $1(\mathfrak{a}, \mathrm{m})=1^{x_{i}(\mathfrak{g})} \neq 1$, we have

and

$$
\vartheta_{l v}\left[l^{-v} m_{i}, 0\right](q \mid 1(g)) \neq 0
$$

$$
\begin{aligned}
\vartheta_{l^{\nu}}\left[l^{-\nu} \mathrm{m}_{i}, 0\right](q \mid 1(g)) & : \vartheta_{l}[0,0](q \mid 1(g)) \\
& \neq \vartheta_{l}\left[l^{-\nu} \mathrm{m}_{i}, 0\right](q \mid 1): \vartheta_{l \nu}[0,0](q \mid 1) .
\end{aligned}
$$

4.2. Let $f_{1}, \ldots, f_{N}$ be abelian functions such that $\Omega\left(f_{1}, f_{2}, \ldots, f_{N}\right)=K_{s, q}$ and $\varphi_{0}, \ldots, \varphi_{N}$ be the theta functions of certain tyer, say $(n, \chi)$, with coefficients in $\Omega$ such that $f_{1}=\varphi_{1} / \varphi_{0}, \ldots, f_{N}=\varphi_{N} / \varphi_{0}$. Putting $\varphi_{i}(u)=\varphi_{i}\left(\mathcal{X}^{-1} u\right) \quad(0 \leq i$ $\leq N)$ and $g_{i}(u)=\psi_{i}(u) / \psi_{0}(u) \quad(1 \leq i \leq N)$, we have a system of abelian functions 
$g_{1}, \ldots, g_{N}$ with coefficients in $\Omega$ such that $g_{i}(\mathcal{X} u)=f_{i}(u)(1 \leq i \leq N)$. Therefore by virtue of Theorems 2 and Lemma 2 we have $\Omega\left(g_{1}(u), \ldots, g_{N}(u)\right)=\Omega\left(f_{1}\left(\chi_{u}\right)\right.$, $\left.\left.\ldots, f_{N}\left(\chi_{u}\right)\right)=\Omega\left(f_{1}(u), \ldots, f_{N}(u), f_{1}(\chi), \ldots, f_{N}(\chi)\right)=\Omega\left(f_{1}(u), \ldots, f_{N}(u)\right)\right)$ $=K_{s, q}$. Hence, by virtue of (18), we get

THEORM 4. There exists a positive integer $n$ such that

$$
\vartheta_{n}\left[g_{2}, 0\right](q \mid u) / \vartheta_{n}[0,0](q \mid u), \ldots, \vartheta_{n}\left[g_{n^{r}}, 0\right](q \mid u) / \vartheta_{n}[0,0](q \mid u)
$$

generate $K_{S, q}$ over $\Omega$, where $\left\{g_{1}=0, g_{2}, \ldots, g_{n^{r}}\right\}$ is a system of representatives of $n^{-1} S^{-1} \mathfrak{M} / S^{-1} \mathfrak{M}$ in $n^{-1} S^{-1} \mathfrak{M}$.

We shall denote by $A_{n, s, q}$ the locus of $\left(\vartheta_{n}\left[g_{1}, 0\right](q \mid u), \ldots, \vartheta_{n}\left[g_{n^{r}}, 0\right](q \mid u)\right)$ over $\Omega$ in the projective space of dimension $n^{r}-1$. The mapping $\rho_{n}: \chi \rightarrow\left(\vartheta_{n}\left[\boldsymbol{g}_{1}\right.\right.$, $\left.0](q \mid \chi), \ldots, \vartheta_{n}\left[g_{n^{r}}, 0\right](q \mid \chi)\right)$ of $\left(\mathbb{K}(\mathrm{K})\right.$ into $A_{n, s, q}$ induces a mapping $\bar{\rho}_{n}$ of $\mathfrak{I}_{s, q}$ into $A_{n, s, q}: \rho_{n}$ is defined on the subset $\{\% \mid$ theta functions of type $(n, \chi)$ do not vanish simultaneously at $\chi_{0}$.

Let us show that $A_{n, s, q}$ has a normal law of composition for a sufficient large $n$.

Proposition 13. If $m$ is a positive integer not less than the positive integer $n$ in Theorem 4. Then $A_{m, s, q}$ has the normal law of composition such that $\rho_{m}(v w)=\rho_{m}(v) \circ \rho_{m}(w)$ for independent $v, w$ in $(\mathrm{K}(\mathrm{K})$ over $\Omega$.

Proof. We shall denote briefly

$$
F_{i-1}(u)=\vartheta_{n}\left[g_{i}, 0\right](q \mid u) / \vartheta_{n}\left[g_{1}, 0\right](q \mid u), \quad\left(2 \leq i \leq n^{r}\right) .
$$

For any independent $v$ and $w$ in $\mathbb{E}(\mathrm{K})$ over $\Omega$ we have, by virtue of Theorem 2 , $F_{i}(v w) \in \Omega\left(F_{1}(v), \ldots, F_{n^{r}-1}(v), F_{1}(w), \ldots, F_{n^{r-1}}(w)\right)$ and $F_{i}\left(v^{-1}\right) \in \Omega\left(F_{1}(v)\right.$, $\left.\ldots F_{n^{r-1}}(v)\right)$. This shows that the loci of $\left(\rho_{m}(v), \rho_{m}(w), \rho_{m}(v w)\right)$ and $\left(\rho_{m}(v)\right.$, $\left.\rho_{m}\left(v^{-1}\right)\right)$ over $\Omega$ define rational mappings $\gamma$ of $A_{m, s, q} \times A_{m, s, q}$ and $\omega$ of $A_{m, s, q}$ onto $A_{m, s, q}$ such that $\gamma\left(\rho_{m}(v), \rho_{m}(w)\right)=\rho_{m}(v w), \omega\left(\rho_{m}(v)\right)=\rho_{m}\left(v^{-1}\right)$. Since $v(w \chi)=(v w) \%, v v^{-1}=1$ and $v w=w v$, we have $\gamma\left(\rho_{m}(v), \gamma\left(\rho_{m}(w), \rho_{m}(\chi)\right)\right)=$ $\gamma\left(\gamma\left(\rho_{m}(v), \rho_{m}(w)\right), \rho_{m}(\chi)\right), \quad \gamma\left(\rho_{m}(v), \omega\left(\rho_{m}(v)\right)\right)=\rho_{m}(1)$ and $\gamma\left(\rho_{m}(v), \rho_{m}(w)\right)$ $=\gamma\left(\rho_{m}(w), \rho_{m}(u)\right)$. Moreover we have $\Omega\left(\rho_{m}(v w), \rho_{n}(w)\right) \subset \Omega\left(\rho_{m}(v), \rho_{m}(w)\right)$ $\subset \Omega\left(\rho_{m}(v), \rho_{m}\left(w v v^{-1}\right)\right)=\Omega\left(\rho_{m}(v), \rho_{m}(v w)\right)$. Similarly we have $\Omega\left(\rho_{m}(w), \rho_{m}(v w)\right)$ $=\Omega\left(\rho_{m}(v), \rho_{m}(w)\right)$. This shows that, putting $\rho_{m}(v) \circ \rho_{m}(w)=r\left(\rho_{m}(v), \rho_{m}(w)\right)$, we have a normal law of composition such that $\rho_{m}(v) \circ \rho_{m}(w)=\rho_{m}(v w)$ and 
$\rho_{m}\left(v^{-1}\right) \circ \rho_{m}(v)=\rho_{m}(1)$.

By virtue of the fundamental theorem of pre-group variety, ${ }^{2)}$ there exists a uniquely determined commutative group variety $A_{m, s, q}^{*}$ such that there exists the birational transformation $\tau_{m}$ of $A_{m, s, q}$ generically surjective on $A_{m, s, q}^{*}$ and the law of composition of $A_{m, s, q}^{*}$ is obtained by transformering of that of $A_{m, s, q}$ by $\tau_{m}$. For $m, m \geq n$, the varieties $A_{m, s, q}^{*}$ and $A_{n, s, q}^{*}$ are isomorphic over $\Omega$ so that we may denote it by $A_{s, q}$. We denote further by $A_{s, q}(\Omega)$ the group consisting of all $\Omega$-rational points on $A_{s, q}$.

PROPOSITION 14. There exists a homomorphism $\bar{\rho}$ of $T_{S, q}$ into $A_{s, q}$ such that $\bar{\rho}(\bar{v})=\left(\tau_{m}^{\circ} \rho_{m}\right)(v)$ for $m \geq n$ and the variable $v$, where $n$ is the integer in Theorem 4 and $\bar{v}$ is the class of $v$ in $\mathfrak{I}_{s, q}$. Moreover we kave $\bar{\rho}\left(\mathfrak{I}_{s, q}(\Omega)\right.$ ) $\subset A_{s, q}(\Omega)$.

Proof. Let $\%$ be any element in $E(\mathrm{~K})$ and $u^{\prime}$ be a variable element in $(E(\mathrm{~K})$ over $\Omega((\chi))$. Then $\rho_{m}(w)$ and $\rho_{m}\left(w^{-1} \%\right)$ are generic points of $A_{m, s, q}$ over $\Omega$. So the birational transformation of $A_{m, s, q}$ onto $A_{s, q}$ is biregular at $\rho_{m}(w)$ and $\rho_{m}\left(w^{-1} \chi\right)$. Hence we may put

(19) $\bar{\rho}(\bar{\chi})=\left(\tau_{m}^{\circ} \mu_{m}\right)(w)+\left(\tau_{m}^{\circ} \rho_{m}\right)\left(w^{-1} \chi\right)$.

Let $w_{1}$ be a variable element in $\left(\xi(\mathrm{K})\right.$ over $\Omega((\chi, w))$. Then since $\rho_{m}(w)$ ${ }^{\circ} \omega\left(\rho_{m}\left(w_{1}\right)\right)=\rho_{m}\left(w w_{1}^{-1}\right)$ and $\rho_{m}\left(w^{-1} \%\right) \circ \omega\left(\rho_{m}\left(w_{1}^{-1} \chi\right)\right)=\rho_{m}\left(w^{-1} \chi\left(w_{1}^{-1} \chi\right)^{-1}\right)$, we have

$$
\begin{aligned}
\left\{\left(\tau_{m} \circ \rho_{m}\right)(w)+\right. & \left.\left(\tau_{m} \circ \rho_{m}\right)\left(w_{1}^{-1} \chi\right)\right\} \\
& -\left\{\left(\tau_{m} \circ \rho_{m}\right)\left(w_{1}\right)+\left(\tau_{m} \circ \rho_{m}\right)\left(w_{1}^{-1} \chi\right)\right\}=0,
\end{aligned}
$$

where $\omega$ is the rational mapping such that $\rho_{m}(v) \circ \omega\left(\rho_{m}(v)\right)=\rho_{m}(1)$.

This shows that $\bar{\rho}(\bar{\chi})$ does not depend on the choice of $w$ and $\bar{\rho}(\bar{\chi})$ is a $\Omega((\%))$-rational point of $A_{s, q}$. Let $\psi_{1}, \chi_{2}$ be elements in $\left(\xi(\mathrm{K})\right.$ and $w_{1}, w_{2}$ be independent elements in $E(K)$ over $\Omega\left(\left(\chi_{1}, \chi_{2}\right)\right)$. Then, since $\rho_{m}\left(w_{1}\right), \rho_{m}\left(w_{2}\right)$ are independent generic points of $A_{m, s, q}$ over $\Omega$ and $\rho_{m}\left(\chi_{1} w_{1}^{-1}\right), \rho_{m}\left(\chi_{2} w_{2}^{-1}\right)$ are independent generic points of $A_{m, s, q}$ over $\Omega$, we have

$$
\begin{aligned}
\bar{\rho}\left(\bar{\gamma}_{1}\right)+\bar{\rho}\left(\bar{\gamma}_{2}\right) & =\left(\tau_{m} \circ \rho_{m}\right)\left(w_{1}\right)+\left(\tau_{m} \circ \rho_{m}\right)\left(w_{1}^{-1} \gamma_{1}\right)+\left(\tau_{m} \circ \rho_{m}\right)\left(w_{2}\right)+\left(\tau_{m} \circ \rho_{m}\right)\left(w_{2}^{-1} \chi_{2}\right) \\
& =\left(\tau_{m} \circ \rho_{m}\right)\left(w_{1} w_{2}\right)+\left(\tau_{m} \circ \rho_{m}\right)\left(\left(w_{1} w_{2}\right)^{-1} \chi_{1} \chi_{2}\right)=\bar{\rho}\left(\bar{\chi}_{1} \bar{\gamma}_{2}\right)
\end{aligned}
$$

and

2) See [3] p. 438, Theorem (Chevalley), or [4] III $7^{\circ}$, p. 50. 


$$
\begin{aligned}
-\bar{\rho}\left(\bar{\chi}_{1}\right) & =-\left(\tau_{m} \circ \rho_{m}\right)(w)-\left(\tau_{m} \circ \rho_{m}\right)\left(w^{-1} \chi_{1}\right) \\
& =\left(\tau_{m} \circ \rho_{m}\right)\left(w^{-1}\right)+\left(\tau_{m} \circ \rho_{m}\right)\left(w^{-1} \chi_{1}\right)=\mu\left(\chi_{1}^{-1}\right) .
\end{aligned}
$$

This proves that $\bar{\rho}$ is a homomorphism of $\mathfrak{I}_{S, q}$ into $A_{s, q}$.

We denote by $\mathfrak{I}_{S, q}^{*}$ the subgronp of $\mathfrak{I}_{S, q}$ consisting of all elements of finite order and denote by $\rho$ the homomorphism of $\left(5(\mathrm{~K})\right.$ into $A_{s, q}$ such that $\rho(v)$ $=\bar{\rho}(\bar{v})$.

PROPOSITION 15. The restriction of $\bar{\rho}$ on $\mathfrak{I}_{s, q}^{*}$ is an isomorphism.

Proof. Let $\%$ be an element of $\mathbb{E}(\mathrm{K})$ such that $\% \in \mathfrak{I}_{S, q}^{*} \Gamma, \bar{\rho}^{-1}(0)$. Since $\mathfrak{I}_{S, q}^{*}$ is the image of $\left\{q(\mathfrak{g}) 1(\mathfrak{h}) ! \mathfrak{g}, \mathfrak{h} \in \mathfrak{M}_{\mathrm{Q}}\right\}$ in $\mathfrak{I}_{s, q}$, there exist $n, \mathfrak{g}, \mathfrak{h}$ such that $\varkappa=q(\mathfrak{g}) 1(\mathfrak{h})$ and $\mathfrak{g}, \mathfrak{h} \in n^{-1} S^{-1} \mathfrak{M}$. It is sufficient to prove $\rho(\mathcal{K}) \neq \rho(w)$ for a variable element $w$ in $\left(\xi(K)\right.$ over $\Omega$. If $\mathfrak{g} \notin S^{-1} \mathfrak{M}$ or $1(\mathfrak{h}) \neq 1$, there exists by Lemmas 6 and 7 a theta function $\varphi(u)$ of some type, say $\left(n^{2}, 1\right)$, with coefficients in $\Omega$ such that $\varphi(1) / \vartheta_{n}:[0,0](q \mid 1) \neq \varphi(q(g) 1(\mathfrak{h})) / \vartheta_{n}:[0,0](q \mid g(\mathfrak{g}) 1(\mathfrak{h}))$. This shows $\varphi(w) / \vartheta_{n}[0,0](q \mid w) \neq \varphi(\% w) / \vartheta_{n}[0,0](q \mid \% w)$. Hence $\rho_{n} 2(w)$ $\neq \rho_{n} \nu(\% w)$. Since $\rho_{n} \nu(w), \rho_{n} \nu(\% w)$ are generic points of $A_{n^{\nu}, s, q}$ over $\Omega, \rho(w)$ $=\left(\tau_{n} \vee \rho_{n} \nu\right)(w) \neq\left(\tau_{n} \nu \circ \rho_{n}\right)(\% w)=\rho(w \%)$. This proves Proposition 15.

THEOREM 5. As, $A_{s}$ is an abelian variety defined over $\Omega$. If $p \neq 0$, the number $\left[A_{s, q}(p):\{0\}\right]$ of $p$-division points of $A_{s, q}$ is $p^{r}$. Let $\rho^{*}$ be the mapping of the field of rational fnnction $\Omega\left(A_{s, q}\right)$ over $\Omega$ into $K_{s, q}$ such that $\rho^{*}(f)(v)=f(\rho(v))$, $\left(f \in \Omega\left(A_{s, q}\right)\right)$. Then $\rho^{*}$ is an isomorphism of the field of rational functions $\Omega\left(A_{s, q}\right)$ onto $K_{s, q}$ such that $\rho^{*}(f)(v w)=f(\rho(v)+\rho(w))$ and $\rho^{*}(f)\left(v^{-1}\right)$ $=f(-\rho(v))$.

Proof. By virtue of the structure theorem on group variety, there exists a connected linear group $L$ defined over $\Omega$ such that $\bar{A}=A_{s, q} / L$ is an abelian variety and $L$ is the direct product of the semi-simple part $L_{s}$ and the unipotent part $L_{l l}{ }^{3)}$ If $l$ is a prime number different from $p$, then the group $L_{s}(l)$ (consisting of all $l$-division points in $L_{s}$ ) contains $l^{\mathrm{dim} L}$ elements and $L_{u}$ has no $l$-divison point other than the unit element. On the other hand, for an abelian variety $A$ we have $[\bar{A}(l):\{0\}]=l^{2 \operatorname{dim} \bar{A}}$ and $[\bar{A}(p):\{0\}] \leqq p^{\mathrm{dim} \bar{A}}$. ${ }^{2}$ On the other hand, by virtue of Proposition 15, $\left[A_{S, q}(l):\{0\}\right]=l^{2 r}$. This shows that the

3) See [3], or [4] III $7^{\circ}$, p. 50 .

4) See [2] Chap. VII. 
linear group $L$ must be the identity group. Namely $A_{s, q}$ is an abelian variety. Therefore by virtue of Proposition 15, $[A(p):\{0\}]=p^{r}$. For sufficient large $m A_{m, s, q}$ is birational equivalent with $A_{s, q}$ and $A_{m, s, q}$ is the locus of ( $\vartheta_{m}\left[g_{1}\right.$, $\left.0](q \mid u), \ldots, \vartheta_{m}\left[g_{m^{r}}, 0\right](q \mid u)\right)$ over $\Omega$. So $\rho^{*}$ is a isomorphism of $\Omega\left(A_{s, q}\right)$ onto $K_{s, q}$. Since $\rho$ is a homomorphism of $\mathbb{E}(\mathrm{K})$ into $A_{s, q}, \rho^{*}$ satisfies the condition in Theorem 5 .

THEOREM 6. There exists a positive integer $n_{0}$ such that for every $n \geq n_{0}$ the locus of

$$
\left(\vartheta_{n}\left[g_{1}, 0\right](q \mid u), \ldots, \vartheta_{n}\left[g_{n^{r}}, 0\right](q \mid u)\right)
$$

over $\Omega$ is a projective embedding of $A_{s, q}$ over $\Omega$.

Proof. An abelian variety defined over $\Omega$ is always embedded in a projective space over $\Omega^{5)} \quad$ Let $\left(f_{0}, \ldots, f_{N}\right)$ be a system of rational functions on $A_{s, q}$ over $\Omega$ such that, if $x$ is a generic point of $A_{s, q}$ over $\Omega,\left(f_{0}(x), \ldots, f_{N}(x)\right)$ is a generic point of the embedding of $A_{s, q}$ in the projective space. Then there exist a positive integer $n$, a mulutiplicative function $\chi$ on $\mathfrak{M}_{Q}$ valued in $\Omega$ and theta functions $\varphi_{0}, \ldots, \varphi_{N+1}$ of type $(n, \chi)$ with coefficients in $\Omega$ such that

$$
f(\rho(u))=\varphi_{i}(u) / \varphi_{r+1}(u) \quad(0 \leq i \leq N) .
$$

This shows that the locus of $\left(\varphi_{0}(u), \ldots, \varphi_{N}(u)\right)$ over $\Omega$ is a projective embedding of $A_{s, q}$ ever $\Omega$. From (18) we know that the locus of

$$
\left(\vartheta_{n}\left[g_{1}, 0\right](q \mid \chi u), \ldots, \vartheta_{n}\left[g_{n^{r}}, 0\right](q \mid \chi u)\right)
$$

over $\Omega$ is a projective embedding of $A_{s, q}$ over $\Omega$. Since $A_{s, q}$ is an abelian variety, $\left(\vartheta_{n}\left[g_{1}, 0\right](q \mid u), \ldots, \vartheta_{n}\left[g_{n^{r}}, 0\right](q \mid u)\right)$ gives also projective embedding of $A_{s, a}$. By virtue of Lemma 2, the embedding is defined over $\Omega$.

4.3. Let us now show that any multiplication $\alpha$ of $q$ induces an endomorphism of $A_{s, q}$. In the classical case, for some special value of $q, A_{s, q}$ has endomorphisms other than those corresponding to the multiplications of $\left(S^{-1}, q\right)$. For non-archimedean case, however, we have no knowledge about such endomorphisms of $A_{s, q}$.

TheOREM 7. There exists an isomorphism $r$ of $\mathfrak{A}\left(\mathrm{S}^{-1}, q\right)$ into the ring of endomorphisms of $A_{s, q}$ such that $\bar{\rho}\left(\bar{\xi}^{\alpha}\right)=\gamma(\alpha) \bar{\rho}(\bar{\xi})\left(\xi \in\left(\xi(\mathrm{K}), \alpha \in \mathfrak{A}\left(S^{-1}, q\right)\right)\right.$,

5) See [6]. 
where $\bar{\rho}$ is the canonical homomorphism of $\mathbb{I}_{s, q}=\left(\mathrm{K}(\mathrm{K}) /\left(\mathfrak{E}\left(S^{-1}, q\right)\right.\right.$ into $A_{s, q}$.

Proof. Let $\varphi_{0}, \ldots, \varphi_{N}$ be a system of theta functions of some type, say $(n, \gamma)$, with coefficients in $\Omega$ such that the locus of $\left(\varphi_{0}, \ldots, \varphi_{N}\right)$ over $\Omega$ is a projective embedding of $A_{s, q}$. Let $\alpha$ be an element of $\mathfrak{A}\left(S^{-1}, q\right)$ and put $\psi_{\alpha, i}(u)$ $=\varphi_{i}\left(u^{\alpha}\right)(0 \leq i \leq N)$. Then, by virtue of Proposition 11, $\psi_{\alpha, i}(u)(0 \leq i \leq N)$ are theta functions of type $\left(n \alpha \alpha^{*}, \chi\right)$ with coefficients in $\Omega$. Hence $\phi_{\alpha, i}(u) / \psi_{\alpha, 0}(u)$ $\in K_{S, q}(1 \leq i \leq N)$. This shows that the locus of

$$
\left(\varphi_{0}(u), \ldots, \varphi_{N}(u)\right) \times\left(\varphi_{0}\left(u^{\alpha}\right), \ldots, \varphi_{N}\left(u^{\alpha}\right)\right)
$$

over $\Omega$ gives a rational mapping $\gamma(\alpha)$ of $A_{s, q}$ into $A_{s, q}$. Since $A_{s, q}$ is an abelian variety, $\gamma(\alpha)$ is defined everywhere on $A_{s, q}$. Furthermore, since

$$
\left(\varphi_{0}\left((u v)^{\alpha}\right), \ldots, \varphi_{N}\left((u v)^{\alpha}\right)\right)=\left(\varphi_{0}\left(u^{\alpha} v^{\alpha}\right), \ldots, \varphi_{N}\left(u^{\alpha} v^{\alpha}\right)\right), \gamma(\alpha)
$$

is an endomorphism of $A_{S, q}$ such that $\rho\left(\xi^{\alpha}\right)=\gamma(\alpha) \rho(\xi)(\xi \in \mathbb{E}(\mathrm{K})) . \quad \gamma$ is an isomorphism, because $\xi^{\alpha} \in \xi\left(S^{-1}, q\right)$ for every $\xi$ in $\xi(\Omega)$ if and only if $\alpha=0$.

Since the group $\mathfrak{I}_{s, q}^{*}$ is isomorphic to the group consisting of all the points of finite order in $A_{s, q}$ by mean of the isomorphism $\bar{\rho}$. So we may choose a system of $l$-adic (resp. $p$-adic) coordinates on $A_{s, q}$ as follows:

$$
\rho(q(\mathfrak{g}) 1(\mathfrak{h})) \rightarrow\left(x_{1}(\mathfrak{g}), \ldots, x_{r}(\mathfrak{g}), x_{1}(\mathfrak{h}), \ldots, x_{r}(\mathfrak{h})\right)(\bmod 1)
$$

$\left(\right.$ resp. $\left.\rho(g(g)) \rightarrow\left(x_{1}(g), \ldots, x_{r}(g)\right)(\bmod 1)\right)$, where $g=\sum_{i=1}^{r} x_{i}(g) m_{i}$ and $\mathfrak{h}=\sum_{i=1}^{r} x_{i}(\mathfrak{h}) m_{i}$

Therefore, by virtue of Theorem 7 , we have

THEOREM 8. There exists a system of l-adic (resp. p-adic) coordimttes on $A_{s, q}$ such that, if we denote by $M_{l}$ (resp. $M_{p}$ ) the l-adic (resp. p-adic) respresentation of the ring of endomorphisms End $\left(A_{s, q}\right)$ of $A_{s, q}$ with respects to the system of coordinates, then

$$
M_{l}(\gamma(\alpha))=\left(\begin{array}{cc}
S \alpha S^{-1} & 0 \\
0 & S \alpha S^{-1},
\end{array}\right)
$$

and, if $p \neq 0$,

$$
M_{p}(\gamma(\alpha))=S \alpha S^{-1} \quad\left(\alpha \in \mathfrak{A}\left(S^{-1}, q\right)\right)
$$

where we identify $\alpha$ with the integral matrix that is the representation of $\alpha$ 
with respect to the coordinate system of $\mathfrak{M}_{\mathrm{Q}}$.

4.4. Let us now prove the existence of a positive symmetric bimultiplicative function $q$ on $\mathfrak{M}_{\mathrm{Q}} \times \mathfrak{M}_{\mathrm{Q}}$ valued in $\Omega^{\times}$such that $\mathfrak{A}\left(S^{-1}, q\right)=\Lambda$.

Propositron 16. Let $\left(\beta_{1}, \ldots, \beta_{h}\right)$ be a (minimal) Q-base of the module of symmetric elements of the commutor algebra $\Lambda_{\mathrm{Q}}^{c}$ of $\Lambda_{\mathrm{Q}}$ in $\operatorname{End}_{\mathrm{Q}}\left(\mathfrak{M}_{\mathrm{Q}}\right)$. Put $S^{-1} \beta_{l}=\left(c_{i j}^{(l)}\right)$ with components $c_{i j}^{(l)}(1 \leq i, j \leq r ; 1 \leq l \leq h)$. Let $q_{0}$ be a positive symmetric bimultiplicative function on $\mathfrak{M}_{\mathrm{Q}} \times \mathfrak{M}_{\mathrm{Q}}$ valued in $\Omega^{\times}$and $\varepsilon_{1}, \ldots, \varepsilon_{h}$ be elements in $\Omega^{\times}$satisfying $v\left(\varepsilon_{1}\right)=\cdots=v\left(\varepsilon_{h}\right)=0$. If $\Lambda_{Q}^{c}$ is generated by $\left\{\beta_{1}, \ldots, \beta_{h}\right\}$ and there exists no relation: $q_{0}(m, n)=\prod_{l=1}^{h} \varepsilon_{l}^{\nu_{l}}\left(\mathfrak{m}, \mathfrak{n} \neq 0\right.$ in $\mathfrak{M}_{Q}$; $\left.\left(\nu_{1}, \ldots, \nu_{h}\right) \neq(0, \ldots, 0)\right)$, then the bimultiplicative function $q$ defined by $q\left(\mathrm{~m}_{i}, \mathrm{~m}_{j}\right)=q_{0}\left(\mathrm{~m}_{i}, \mathrm{~m}_{j}\left(\prod_{i=1}^{h} \varepsilon_{l}^{c_{i j}^{(i)}}(1 \leq i \leq j \leq r)\right.\right.$ is a positive symmetric multiplicative function such that $\mathfrak{A}\left(S^{-1}, q\right)=A$.

Proof. Since $\beta_{l}^{*}=S^{t} \beta_{l} S^{-1}=\beta_{l}$, we have $c_{i j}^{(l)}=c_{i j}^{(l)}(1 \leq i, j \leq r ; 1 \leq l \leq h)$. By the positivity of $q_{0}$ and $v(q(\mathfrak{m}, \mathfrak{n}))=v\left(g_{0}(m, \mathfrak{n})\right)\left(\mathfrak{m}, \mathfrak{n} \in \mathfrak{M}_{Q}\right), q$ is a positive symmetric bimultiplicative function. Let $\alpha$ be any element in $\operatorname{End}_{Q}\left(\mathfrak{M}_{Q}\right)$ such that $\alpha S^{-1} \mathfrak{R} \subset S^{-1} \mathfrak{M}$ and put $\alpha \mathrm{m}_{i}=\sum_{j=1}^{r} a_{i j}(\alpha) \mathrm{m}_{j}$ with $a_{i j}(\alpha)$ in $Z(1 \leq i, j \leq r)$. Then, since $m_{1}=S^{-1 t}(1,0, \ldots, 0), \ldots, m_{r}=S^{-1 t}(0, \ldots, 0,1)$, we have $\left(a_{i j}(\alpha)\right)$ $=S^{-1} \alpha S$ and $\left(a_{i j}\left(\alpha^{*}\right)\right)=S^{-1} \alpha^{*} S=S^{-1}\left(S^{t} \alpha S^{-1}\right) S={ }^{t} \alpha$. Let us now write the condition on $\left(a_{i j}(\alpha)\right)$ to be a multiplication of $\left(S^{-1}, q\right): q\left(\alpha m_{i}, m_{j}\right)=\prod_{l} q\left(m_{l}\right.$, $\left.\mathrm{m}_{j}\right)^{a_{i l(\alpha)}}=q\left(\mathrm{~m}_{i}, \alpha^{*} \mathrm{~m}_{j}\right)=\prod_{l} g\left(\mathrm{~m}_{i}, \mathrm{~m}_{l}\right)^{a_{j l}\left(\alpha^{*}\right)}(1 \leq i, j \leq r)$. Hence $\alpha \in \mathfrak{H}\left(S^{-1}, q\right)$ if and only if $\prod_{l} q_{0}\left(m_{i}, m_{l}\right)^{a_{j l}\left(\alpha^{*}\right)} \prod_{l, s} \varepsilon_{s}^{c_{i l}^{(s)} a_{j l}\left(\alpha^{*}\right)}=\prod_{l} q_{0}\left(m_{l}, m_{j}\right)^{a_{i l}(\alpha)} \prod_{l, s} \varepsilon_{s}^{a_{i l} c_{l j}^{(s)}}$. Since there exists no relation $g_{0}(m, \mathfrak{n})=\prod_{l} \varepsilon_{l}^{\nu_{l}}\left(\left(\nu_{1}, \ldots, \nu_{h}\right) \neq(0, \ldots, 0)\right)$. we see that $\alpha \in \mathfrak{A}\left(S^{-1}, q\right)$ if and only if $(*)\left(a_{i l}(\alpha)\right)\left(c_{l j}^{(s)}\right)=\left(c_{i l}^{(s)}\right)^{t}\left(a_{l j}\left(\alpha^{*}\right)\right)(1 \leq s \leq h)$.

Since $\left(a_{i j}(\alpha)\right)=S^{-1} \alpha S,\left(a_{i j}\left(\alpha^{*}\right)\right)={ }^{t} \alpha$ and $\left(c_{i j}^{(s)}\right)=S^{-1} \beta_{s}$, the condition $(*)$ is equivalent to $\alpha \beta_{s}=\beta_{s} \alpha(1 \leq s \leq h)$. On the other hand $A_{\mathrm{Q}}$ is semi-simple, the commutor algebra of $\Lambda_{\mathrm{Q}}^{c}$ coincides with $\Lambda_{\mathrm{Q}}$. Therefore, by virtue of the assumption on $A_{\mathrm{Q}}^{c}$, the condition $(*)$ is equivalent to $\alpha$ belongs to $A_{\mathrm{Q}}$. This proves Proposition 16.

Proposition 17. If $\Lambda_{Q}^{c}$ is generated by symmetric elements in $\Lambda_{Q}^{c}$, then there exists a positive symmetric bimultiplicative function $q$ on $\mathfrak{M}_{Q} \times \mathfrak{M}_{Q}$ valued in $\Omega^{\times}$ such that $\mathfrak{A}\left(S^{-1}, q\right)=\Lambda$. 
Proof. Since $\Omega$ is $v$-complete, $\Omega$ contains infinite many elements $\xi_{1}, \xi_{2}, \ldots$ such that $v\left(\xi_{i}\right)=0(i=1,2, \ldots)$ and $\xi_{1}, \xi_{2}, \ldots$ are algebraically independent over the prime field $I$ of $\Omega$. Let $q_{0}$ be a positive symmetric bimultiplicative function on $\mathfrak{M}_{\mathrm{Q}} \times \mathfrak{M}_{\mathrm{Q}}$ valued in $\Omega^{\times}$. Then $q_{0}(\mathfrak{m}, \mathfrak{n})\left(\mathfrak{m}, \mathfrak{n} \in \mathfrak{M}_{\mathrm{Q}}\right)$ are algebraic over $g_{0}\left(m_{i}, m_{j}\right)(1 \leq i \leq j \leq r)$, so that there exist $\xi_{i_{1}}, \ldots, \xi_{i_{h}}$ in $\Omega^{\times}$such that $v\left(\xi_{i_{1}}\right)=\cdots=v\left(\xi_{i_{h}}\right)=0$ and $\xi_{i_{1}}, \ldots, \xi_{i_{h}}$ are algebraioally independent over $\Pi\left(\left\{q_{0}(\mathfrak{m}, \mathfrak{n}) \mid \mathfrak{m}, \mathfrak{n} \in \mathfrak{M}_{\mathrm{Q}}\right\}\right)$. This means that there exists no relation $q_{0}(\mathfrak{m}, \mathfrak{n})$ $=\prod_{l} \xi_{i l}^{\nu}{ }^{l}\left(\left(\nu_{1}, \ldots, \nu_{h}\right) \neq(0, \ldots, 0)\right)$. Hence, by virtue of Proposition 16, putting $q\left(\mathrm{~m}_{i}, \mathrm{~m}_{j}\right)=q_{0}\left(\mathrm{~m}_{i}, \mathrm{~m}_{j}\right) \prod_{l} \xi_{i l^{\prime}}^{(l)}(1 \leq i \leq j \leq \boldsymbol{r})$, we have $\mathfrak{\imath}\left(S^{-1}, q\right)=\Lambda$.

\section{BIBLIOGRAPHY}

[1] A. Albert, On involutorial algebras, Proc. Nat. Acad. Sci. U.S.A. Vol. 4, No. 7 (1955), p. $480-482$.

[2] S. Lang, Abelian varieties, (1959).

[3] M. Rosenlicht, Basic theorems on algebraic groups, Amer. J. Math. Vol. 78, No. 2 (1956), p. 401-443.

[4] P. Serre, Groupes algébriques et corpes de classes, 1959 (Paris).

[5] A. Weil, On algebraic groups and homogeneous spaces, Amer. J. Math. Vol. 77, No. 3 (1955), p. 493-512.

[6] A. Weil, On the projective embedding of abelian varieties, in the volume in honor of S. Lefschetz, Princeton (1957).

\section{Mathematical Institute}

Nagoya University 